\title{
BRD2 regulation of sigma-2 receptor expression upon cytosolic cholesterol deprivation
}

Hongtao Shen ${ }^{1}$, Jing $\mathrm{Li}^{1}$, Xiujie Xie ${ }^{2}$, Huan Yang ${ }^{1}$, Mengxue Zhang ${ }^{1}$, Bowen Wang ${ }^{2}$, K. Craig Kent $^{2}$, Jorge Plutzky ${ }^{3}$, Lian-Wang Guo ${ }^{1,2, *}$

${ }^{1}$ Departments of Surgery and Physiology/Cell Biology, College of Medicine; Davis Heart and Lung Research Institute, Wexner Medical Center. The Ohio State University, Columbus, $\mathrm{OH}$ 43210, USA.

${ }^{2}$ Department of Surgery, College of Medicine; Davis Heart and Lung Research Institute, Wexner Medical Center. The Ohio State University, Columbus, OH 43210, USA.

${ }^{3}$ Cardiovascular Division, Brigham and Women's Hospital, Harvard Medical School, Boston, MA 02115

Running title: BRD2 regulates sigma-2 receptor gene transcription

* Corresponding author:

Lian-Wang Guo, Ph.D.

Department of Surgery and Department of Physiology \& Cell Biology

The Ohio State University, 473 West $12^{\text {th }}$ Ave, 611B DHLRI

Columbus, $\mathrm{OH} 43210$, USA

Tel: +1 614292 5276. Email: lianwang.guo@osumc.edu

Competing Interests: The authors declare no competing interests. 


\section{Abstract}

Traditionally a pharmacologic target for antipsychotic treatment, the sigma-2 receptor (S2R) was recently implicated in cholesterol homeostasis. Here we investigated the transcriptional regulation of S2R by the Bromo/ExtraTerminal epigenetic reader family (BETs, including BRD2, $3,4)$ upon cholesterol perturbation.

Cytosolic cholesterol deprivation was induced using an export blocker of lysosomal cholesterol in ARPE19 cells. This condition upregulated mRNA and protein levels of S2R, and of SREBP2 but not SREBP1, transcription factors key to cholesterol/fatty acid metabolism. Silencing BRD2 but not BRD4 (though widely deemed as a master regulator) or BRD3 prevented S2R upregulation induced by cholesterol deprivation. Silencing SREBP2 but not SREBP1 diminished S2R expression. Furthermore, BRD2 co-immunoprecipitated with the SREBP2 transcriptionactive $\mathrm{N}$-terminal domain, and chromatin immunoprecipitation-qPCR showed a BRD2 occupancy at the S2R gene promoter.

In summary, this study reveals a novel BRD2/SREBP2 cooperative regulation of S2R transcription in response to cytosolic cholesterol deprivation, thus shedding new light on epigenetic control of cholesterol biology.

Keywords: Sigma-2 receptor, transcription, BRD2, SREBP2, cholesterol deprivation 


\section{Introduction}

After decades of studies, cholesterol biology remains inadequately understood, in particular, the regulations involving lysosomes, which distribute cholesterol to other organelles ${ }^{1}$. Recently, TMEM97 was reported as a novel player in cholesterol transport ${ }^{2,3}$. An ER resident protein, TMEM97 can translocate to the lysosomal membrane where it appears to attenuate the activity of NPC1 (Niemann-Pick disease, type C) ${ }^{3}$, the transporter that "pumps" cholesterol out of the lysosome. TMEM97 was also suggested to interact with LDLR thereby involved in cholesterol uptake $^{3}$. Intriguingly, the coding gene of the sigma-2 receptor (S2R), an enigmatic drug binding site pharmacologically identified 40 years ago, was finally (in 2017) unveiled to be TMEM974.

There are a very small number of papers published on TMEM97 (a.k.a. MAC30) ${ }^{5}$; studies on S2R are largely limited to pharmacology such as anti-psychotic treatments ${ }^{6}$. As a result, little is known about the molecular regulations of S2R/ TMEM97/ MAC30 (hereafter denoted as S2R for clarity $)^{4}$. S2R is highly expressed in progressive tumors and thereby targeted as a biomarker for diagnostic imaging ${ }^{7}$. Silencing S2R appeared to alleviate Niemann-Pick disease condition in a mouse model, which features mutated NPC1 and consequential cholesterol accumulation in lysosomes ${ }^{2}$. It is thus important to understand how S2R expression is controlled, whereas little is known at present.

Gene transcription programs are coordinately governed by transcription factors (TFs) and epigenetic factors. Rapidly growing literature supports a notion that the family of BETs (Bromo/ExtraTerminal-domain proteins) act as epigenetic determinants of transcription programs $^{8}$. Among the BETs (BRD2, 3, 4), BRD4 is best studied and thought to function as a lynchpin-organizer of transcription assemblies. While its C-terminal domain (absent in BRD2 and BRD3) interacts with the transcription elongation factor (pTEFb) that activates the RNA polymerase II, its two bromodomains read (bind) acetylation bookmarks on histones and transcription factors. These interactions usher a transcription assembly to specific genomic loci ${ }^{9}$. BRD4 has been shown to play a master role in broad cellular processes ranging from proliferation to differentiation ${ }^{10}$ to autophagy ${ }^{11}$. Curiously, whether BETs are directly involved in cholesterol homeostasis remained unclear ${ }^{12}$. Relevant to this question, recent studies identified a crucial role of BRD4 in adipogenesis ${ }^{10}$. We were thus encouraged to explore whether BRD4 regulates the expression of S2R, a novel modulator of cholesterol transport ${ }^{2}$.

Herein we found that pan-BETs inhibition abolished S2R upregulation that was induced by cytosolic cholesterol deprivation. However, it was BRD2 but not BRD4 or BRD3 that was responsible for this BET function. This was unexpected given that BRD4 has been widely reported to be the determinant BET in diverse processes ${ }^{8}$. We also found that BETs inhibition repressed the transcription of both SREBP1 and SREBP2, the master TFs governing fatty acid and cholesterol homeostasis, and silencing SREBP2 but not SREBP1 inhibited S2R expression. Further results suggested a novel epigenetic mechanism whereby BRD2/SREBP2 co-occupy promoter regions of the S2R gene activating its transcription. Considering that BETs and S2R are targets of clinical (or trial) drugs ${ }^{6,13}$, our findings may impact the studies on epigenetic regulations of cholesterol biology as well as translational medicine. 


\section{Results}

\section{Pan-BETs inhibition represses S2R expression that is stimulated by cytosolic cholesterol deprivation}

To study epigenetic regulations of S2R, we chose ARPE19, a human epithelial cell line where cholesterol plays a critical role in cellular function/dysfunction ${ }^{14}$. To establish a cellular model to monitor S2R level changes, we first tested commonly used cytokine stimulants, including PDGF-AA, PDGF-BB, and TGF $\beta 1$. However, they did not significantly alter S2R mRNA levels (Figure S1). We then tested U188666A (hereafter abbreviated as U18), an NPC1 inhibitor that keeps cholesterol trapped inside the lysosome thereby generating a cholesterol-deprived cytosolic environment. We found that treatment with U18 increased S2R mRNA by up to 8 fold and S2R protein by 2 fold. Interestingly, pretreatment with JQ1 (first-in-class BETs-selective inhibitor) ${ }^{15,16}$ abrogated this U18-induced S2R upregulation (Figure 1, A and C). In contrast to U18 treatment, serum starvation had a relatively minor effect on S2R mRNA and protein upregulation (Figure 1, B and D). Confirming the function of U18 in reducing cytosolic cholesterol, Figure 1E showed that filipin staining of cholesterol was largely absent in the cytosol in U18-treated ARPE19 cells. The staining instead accumulated in perinuclear structures, which are typically known to be lysosomes ${ }^{3}$. By contrast, decrease of cytosolic cholesterol was less prominent under the starvation condition, consistent with a minor effect on S2R upregulation.

Taken together, whereas cytosolic cholesterol deprivation dramatically elevated S2R expression, pan-BETs inhibition with JQ1 abolished this upregulation.

\section{Silencing BRD2 but not BRD4 or BRD3 reduces S2R mRNA and protein}

JQ1 is highly selective to the BET family yet it is a pan inhibitor that blocks the bromodomains in all BETs ${ }^{16}$. We therefore next determined by gene silencing which BET mainly accounted for the U18-induced S2R upregulation. Since there was no previous report to follow for silencing BETs in ARPE19 cells, we first tried lentiviral expression of shRNAs. BRD3 and BRD4 were effectively silenced by their respective shRNAs (Figure 2, A-C), yet BRD2 silencing was inefficient and we therefore used BRD2 siRNA instead. Given a wealth of literature evidence indicating BRD4 as a powerful regulator in a broad range of processes ${ }^{17}$, we expected BRD4 to be the determinant BET. To our surprise, silencing neither BRD4 nor BRD3 reduced U18-induced S2R expression (Figure 2, D-F); rather, silencing BRD2 attenuated U18-induced S2R (mRNA and protein) upregulation. To solidify this conclusion, we used two BRD3 shRNA sequences and two BRD4 shRNA sequences (Figure 2, B and C) (Figure S2) and furthermore, a BRD4 siRNA (Figure 3), but neither reduced S2R expression.

It is noteworthy that BRD4 knockdown appeared to increase BRD2 protein and vice versa though without reaching statistical significance (Figure 3, A-D). An interesting question thus arose as to whether BRD4 knockdown could have raised S2R levels by increasing BRD2. We then silenced BRD4 in addition to BRD2 (Figure 3E), and the decrease of S2R became no longer evident (Figure 3F). The simplest explanation is that the changes of S2R toward opposite directions caused by silencing BRD2 and BRD4 canceled each other. Therefore, this result further supports a role for BRD2 in positively regulating S2R expression.

\section{Silencing SREBP2 but not SREBP1 represses S2R mRNA and protein expression}

In a previously reported RNAi screening study, S2R (TMEM97) was found to be a target gene of the transcription factor SREBP2 ${ }^{3}$. However, detailed studies on S2R transcriptional activation were not available, and whether S2R is under the control of the functionally paired transcription 
factor SREBP1 was not known. We thus performed silencing of SREBP2 and SREBP1 to determine an effect on S2R expression, either under U18 or starvation treatment. As shown in Figure 4A, whereas S2R mRNA levels were elevated by either U18 or starvation (to a lesser extent), SREBP2 silencing diminished this upregulation. By contrast, SREBP1 silencing did not reduce but rather, further enhanced S2R mRNA expression (Figure 4B); this enhancement was paralleled by increased BRD2 mRNA levels. Similar results occurred at the protein level; i.e. SREBP2 silencing abrogated S2R protein upregulation without changing BRD2 protein levels (Figure 4C), however, SREBP1 silencing appeared to increase BRD2 and S2R protein (no significance though) (Figure 4D). Of note, silencing SREBP2 reduced S2R expression without altering BRD2 mRNA or protein levels (Figure 4C), suggesting that SREBP2 is possibly downstream but not upstream of BRD2.

\section{Pan-BETs inhibition suppresses SREBP2 and SREBP1 mRNA levels}

Given that S2R expression was controlled by both BRD2 and SREBP2, an epigenetic factor and a transcription factor, respectively (Figures 1-4), we next asked whether BRD2 regulated S2R expression via SREBP2. As shown in Figure 5 (A-D), treatment with U18 increased SREBP2 mRNA (by $\sim 4$ fold) and protein (albeit statistically insignificant), and SREBP1 mRNA to a minor extent. In contrast to U18, the starvation condition upregulated both SREBP2 and SREBP1 mRNA (by 10-15 fold) and protein levels (Figure 5, E-H). Remarkably, pretreatment with JQ1 abolished all of these changes. Moreover, using a different cell line (HEK293), we also observed upregulation of SREBP2 and S2R in U18-treated cells and its abolishment by pretreatment with JQ1 (Figure 5, I-K). These results suggest that the BETs family control the transcription of SREBP2 and SREBP1, two key TFs in cholesterol and fatty acid metabolism. To the best of our knowledge, BETs epigenetic regulations of SREBPs have not been previously determined in the specific context of cholesterol perturbation.

We then determined whether silencing a BET influences SREBP protein production. Figure 6 shows that neither BRD2 nor BRD4 silencing significantly altered SREBP2 or SREBP1 protein levels. This lack of changes in SREBP protein levels may be rationalized by posttranscriptional regulations.

\section{BRD2 co-immunoprecipitates with the SREBP2 transcription-active N-terminal domain}

Accumulating evidence suggests that BETs cooperate with specific TFs to assume transcriptional activation of select sets of genes, by two possible ways: altering the TF protein level or forming a complex with the TF to activate the transcription of target genes. Since the former did not occur (Figure 6), we next determined whether BRD2 forms a complex with the SREBP2 protein in the regulation of S2R transcription. Indeed, our data indicated that BRD2 coimmunoprecipitated with the SREBP2 N-terminal half molecule (Figure 7A), which is known to translocate into the nucleus to assume the SREBP2 TF function ${ }^{3}$. The specificity of this co-IP is manifested by the lack of BRD2 pulldown in the empty vector control (FLAG-GFP). The specific BRD2/SREBP2(N-term) co-IP was observed either without (Figure 7A) or with (Figure S3) U18 treatment.

\section{BRD2 immunoprecipitates with SREBP-binding DNA regions of the S2R gene promoter}

Finally, we used a BRD2 antibody for IP and performed ChIP-qPCR to detect S2R promoter regions that contain predicted SREBP-binding motifs (sterol response consensus elements) ${ }^{3}$. We found that while U18 treatment increased qPCR signal of a S2R promoter region by $\sim 2$ fold, overexpression of BRD2 further augmented the signal (Figure 7B). Similar results were obtained from experiments detecting other two different S2R gene promoter regions (Figure S4). The specificity of the ChIP-qPCR assay was confirmed by an outstanding signal-to- 
background ratio ( 6-10 fold), i.e. a large difference in qPCR reading between the experiment using a BRD2 antibody for ChIP and that using IgG for control.

In aggregate, these results suggest that BRD2 upregulates S2R expression not by increasing the SREBP2 protein, rather, by forming a BRD2/SREBP2 complex that occupies $\mathrm{S} 2 \mathrm{R}$ gene promoter regions to activate its transcription.

\section{Discussion}

Cholesterol dysregulation leads to a myriad of pathological conditions ${ }^{14}$; S2R was recently suggested as a novel player in cholesterol intracellular transport ${ }^{3}$. To the best of our knowledge, the current report is the first to reveal a BETs-governed epigenetic control of S2R expression. We differentiated that silencing BRD2 but not BRD4 (though widely deemed as a master BET) or BRD3 effectively reduced S2R expression. While pan-BETs inhibition blocked the transcription of both SREBPs, silencing SREBP2 but not SREBP1 repressed S2R expression. Furthermore, our data provided evidence for that BRD2 controls S2R transcription not by increasing the SREBP2 protein but by forming a BRD2/SREBP2 complex at S2R gene promoter regions. Thus, our study suggests a previously unrecognized epigenetic mechanism whereby the duo of BRD2/SREBP2 positively regulate S2R expression in response to cholesterol deprivation in the cytosol.

The finding of BETs-dominated regulation of S2R expression is significant for the following reasons. (1) Epigenetics is crucial in cellular responses to extra- and intra-cellular environmental cues; however, little is known about epigenetic regulations of $\mathrm{S} 2 \mathrm{R}^{18}$, whose expression is highly sensitive to cholesterol level perturbation. This knowledge gap likely stems from the fact that S2R is one of very few drug targets whose coding gene remained unknown until very recently ${ }^{4}$. (2) S2R was implicated (via pharmacology) in neurological disease ${ }^{19}$ (e.g. Alzheimer's) ${ }^{20}$, psychiatric disorders ${ }^{6,21}$, and cancers ${ }^{22}$. In fact, S2R ligands have long been clinically used as antidepressants (e.g. haloperidol) ${ }^{6}$. Moreover, high S2R abundance was found in tumor tissues and cells, as detected with labeled S2R ligands ${ }^{7,22}$ or unknowingly as TMEM97/ MAC30 ${ }^{23}$. As such, S2R is often targeted for cancer imaging (e.g. PET scanning) ${ }^{7}$. More recently, it was reported that S2R (TMEM97) knockdown attenuated Niemann-Pick disease phenotypes in a mouse model, linking S2R to lysosomal cholesterol export ${ }^{2}$. (3) S2R was found to re-locate to lysosomes when intracellular cholesterol levels dropped ${ }^{3}$. It was thus speculated that S2R may interact with NPC1 modulating its cholesterol-exporting function in the lysosomal membrane, whereas with no direct evidence available. (4) Recent studies suggested that S2R co-localizes with LDLR, which via internalization carries esterified cholesterol into the cell ${ }^{3}$; S2R knockdown or knockout impairs cholesterol (and LDLR) uptake. Together, these studies indicated the biological importance of S2R and its regulators and motivated our investigation into BETsdictated epigenetic regulation of S2R expression.

In this novel regulation, it is somewhat surprising that BRD2 rather than BRD4 is found to be the determinant BET. In contrast to BRD2 and BRD3, BRD4 has been intensively studied and shown to play a critical role in many crucial cellular processes and pathological conditions ${ }^{8,24}$. The BRD4 molecule (vs BRD2 and BRD3) has nearly doubled length which contains a unique C-terminal domain. From a functional/structural perspective, BRD4 is dubbed a "Swiss army knife"25. Its two bromodomains "dock" the BRD4-organized regulatory complex (including TFs and cis-regulators) to specific bookmarked chromatin sites, with its C-terminal domain promoting transcriptional activation by interacting with the transcription elongation factor that in 
turn activates the RNA polymerase II. BRD4 was very recently found to possess intrinsic kinase $^{26}$ and acetyl transferase activities ${ }^{27}$. However, in our specific experimental setting of U18induced cytosolic cholesterol deprivation, it was BRD2 but not BRD4 that positively regulated S2R transcription. Inasmuch as BRD2 lacks a C-terminal domain and its bromodomain sequences are different from that of BRD $4^{16}$, our results may implicate a BET mechanism distinct from that of BRD4. Given limited information about BRD2 functional mechanisms, future studies are needed to elucidate the molecular workings that underlie BRD2-dominated epigenetic control of S2R transcription.

To this end, the previous evidence for S2R being a target gene of SREBP2 3 inspired our investigation that led to another novel finding, i.e. BETs govern the transcription of both SREBP1 and SREBP2, the TFs key to fatty acid/cholesterol regulations. This BETs control over SREBP's transcription is exciting, given that BETs and SREBPs are both master regulators of vital cellular activities yet their relationship was previously unknown in the context of cholesterol homeostasis where SREBPs are critically important. Thanks to the recent discovery of inhibitors selective for BETs ${ }^{15,28}$, important BETs functions have been recently identified. BETs inhibition was initially shown to be highly effective in blocking transcription programs of inflammation ${ }^{28}$ and oncogenic proliferation ${ }^{29}$. The importance of BET biology was then extended to stem cell differentiation, hematopoiesis, synaptic plasticity ${ }^{12}$, and recently, also adipogenesis ${ }^{10,30}$. Most recently, BRD4 was found to regulate intra-nuclear/cellular processes as well, such as chromatin architectural remodeling ${ }^{31}$ and autophagy ${ }^{11}$. However, there is a dearth of information on a role for BETs in regulating SREBPs. The closest relevance is a report on BETs-associated super-enhancers formed during adipogenesis ${ }^{10}$. While SREBP1 (but not SREBP2) was on the list of JQ1-regulated genes derived from RNAseq data, the relationship between BETs and SREBP1 was not specifically examined. Increased HDL-cholesterol or decreased LDLcholesterol was observed in animal plasma after treating with a BETs inhibitor ${ }^{32,33}$. However, little is known about specific BETs-dominated cholesterol regulatory pathways, underscoring the importance of investigating BETs regulations of SREBPs and their downstream effector genes.

SREBPs could be reasonably categorized as master TFs. There are total over one thousand TFs, only a limited number of them are deemed master TFs. These master TFs often direct transcription programs that define a cell type or cell state ${ }^{8}$. As such, they are sensitive to extraand intra-cellular environmental perturbations, and their expression levels and activities are key to cell state changes and associated disease conditions ${ }^{34}$. Recently, master TFs were found to co-localize with BRD4 in the genomic landscape thereby playing a critical role in inflammatory (e.g. NFkB) ${ }^{34}$, proliferative (c-Myc) ${ }^{29,35}$, or immunological (T-bet) processes. Another prominent feature of master TFs is that they potently co-activate the transcription of specific sets of genes, by forming a complex with BETs to promote not only target gene expression but also their own transcription $^{8}$. In consonance, herein our ChIP-qPCR data provided evidence for BRD2 occupancy at the SREBP2 gene promoter (Figure S5). Based on these criteria, SREBP2 and SREBP1 appear to be master TFs. SREBP1 and SREBP2 differentially regulate fatty acid and cholesterol pathways (though with possible crosstalk). This may explain our observation that SREBP2 but not SREBP1 positively regulated S2R expression upon cholesterol deprivation. Of note, serum starvation markedly stimulated the expression of both SREBP2 and SREBP1, likely because serum contains both cholesterol and fatty acids. Consistently, pan-BETs inhibition with JQ1 averted upregulation of both SREBP1 and SREBP2 mRNAs stimulated by serum starvation. In addition, JQ1 also reduced mRNAs of SREBP target genes tested herein including not only S2R, but NPC1, NPC2, and LXRs as well (Figure S6). As such, BETs together with SREBPs may govern transcription programs of the cholesterol /fatty acid pathways. For this possibility, future RNAseq studies are needed to provide comprehensive evidence. 
Furthermore, our results suggest that in the condition of cholesterol deprivation the BRD2/SREBP2 duo accounted for the upregulation of S2R. Evidence includes the following: (1) U18 treatment dramatically upregulated S2R and SREBP2 but not SREBP1 expression. (2) Silencing SREBP2 but not SREBP1 reduced S2R mRNA and protein. (3) BRD2 coimmunoprecipitated with the SREBP2 N-terminal TF domain. (4) ChIP-qPCR assays using a BRD2 antibody suggested that BRD2/SREBP2 co-occupied S2R promoter regions, and this cooccupancy was enhanced by U18-induced cholesterol deprivation and BRD2 overexpression. This novel result is consistent with the well-established SREBP2 functional mechanism; i.e. SREBP2 in an inactive state resides in the endoplasmic reticulum (ER) membrane, but upon decrease of cytosolic (hence ER) cholesterol, SREBP2 is transported to Golgi and cleaved into two half molecules ${ }^{3}$. Whereas the $\mathrm{C}$-terminal half stays in the cytosol, the $\mathrm{N}$-terminal half enters the nucleus acting as a TF for the expression of genes involved in cholesterol metabolism and transport. Taken together, the pockets of new information obtained herein and that from the literature form a coherent picture of BRD2/SREBP2-dictated S2R transcription in response to cytosolic cholesterol perturbation.

\section{Conclusions}

We present the first evidence on BETs control over the transcription of S2R, a recently unveiled player in cholesterol-associated regulations. Our results have sketched out a potential epigenetic regulatory mechanism whereby BRD2 forms a complex with SREBP2 that localizes at the S2R gene promoter activating S2R transcription. Further investigation may shed new lights on BRD2-dominated transcription programs that sensitively respond to cholesterol level changes. Along this line, studies on BETs and S2R, both targets of increasing clinical (trial) drugs $^{6,13,24,36,37}$, may synergize interventional opportunities for cholesterol-associated pathological conditions.

\section{Acknowledgments and Sources of Funding.}

This work was supported by NIH R01 grants HL133665 and EY029809 to L.-W.G., HL143469 and HL129785 to K.C.K. and L.-W.G., and an AHA pre-doctoral award 17PRE33670865 (to M.X.Z.). 


\section{Methods}

\section{Major materials}

JQ1 was purchased from Apexbio (A1910). U18666A was from Sigma-Aldrich (662015). Filipin complex was from Sigma-Aldrich (F9765). ARPE19 cells and HEK293 cells were obtained from American Type Culture Collection (ATCC). Scrambled and BRD2-, BRD4-, or SREBP1-specific siRNAs were from Thermo Fisher Scientific (scrambled: AM4635; BRD2: AM16708, ID-118266; BRD4: 4457298, ID-s23902; SREBP1: AM51331, ID-5140). Lipofectamine3000 was from Thermo Fisher Scientific (L3000008).

\section{Cell culture and treatment}

ARPE19 cells were cultured in the DMEM/F12 medium (Thermo Fisher Scientific, 11320082) supplemented with $10 \%$ fetal bovine serum (FBS) and penicillin/streptomycin (Thermo Fisher Scientific, 5140163 ) at $37^{\circ} \mathrm{C}$ in a humidified atmosphere with $5 \% \mathrm{CO}_{2}$. HEK293 cells were maintained in DMEM (Thermo Fisher Scientific, 10569010) supplemented with 10\% FBS and penicillin/streptomycin. To induce cytosolic cholesterol deprivation, ARPE19 cells were seeded in 6 -well plates at $3 \times 10^{5}$ cells/well and cultured for $24 \mathrm{~h}$, U18666A was then added (final $5 \mu \mathrm{M}$ ) and incubated for another $24 \mathrm{~h}$. In some experiments, JQ1 was added $(5 \mu \mathrm{M})$ together with U18666A. For starvation experiments, the culture medium containing 10\% FBS was changed to that without FBS in which cells were cultured for $24 \mathrm{~h}$. Cells were then collected for various analyses. For cholesterol staining, cells were washed 3x with PBS before fixing with $3 \%$ paraformaldehyde for $1 \mathrm{~h}$. The reaction was stopped with glycine $(1.5 \mathrm{mg} / \mathrm{ml})$ and the cells were then stained for $2 \mathrm{~h}$ at room temperature in the filipin working solution $(0.05 \mathrm{mg} / \mathrm{ml}$ in PBS with $10 \%$ FBS). Images were taken with the Nikon fluorescence microscope using a UV filter set (340-380 $\mathrm{nm}$ excitation).

\section{RNA isolation, reverse transcription, and quantitative real-time PCR (qRT-PCR)}

Total RNA was isolated and purified using Trizol Reagent (Thermo Fisher Scientific,15596026) following the manufacturer's instruction. RNA was reverse-transcribed using the High-Capacity cDNA Reverse Transcription kit (Thermo Fisher Scientific, 4368814). cDNA of $1 \mu \mathrm{Lfrom} 20 \mu \mathrm{L}$ reaction volume was amplified by real-time quantitative PCR (Applied Biosystems Quant Studio 3, Thermo Fisher Scientific) with Perfecta SYBR Green Master Fast Mix (VWR, 101414-286) ${ }^{38}$. Relative gene expression was determined by the $2^{-\Delta \Delta \mathrm{Ct}}$ method, normalized to GAPDH, and presented as relative mRNA levels. qPCR analyses were done in triplicate. Experiments were repeated at least twice. Primers are listed in Table S1.

\section{Preparation of lentivectors for shRNA expression}

The pLKO.1-puro empty vector was purchased from Addgene (\#8453). A scrambled shRNA control and gene-specific shRNAs were designed through RNAi Central (http:// cancan.cshl.edu/RNAi_central/step2.cgi). The corresponding oligonucleotides (ordered from Thermo Fisher Scientific) were annealed $\left(95^{\circ} \mathrm{C}\right.$ to $\left.25^{\circ} \mathrm{C}, 0.1^{\circ} \mathrm{C} / \mathrm{s}\right)$ and cloned into the pLKO.1puro vector, followed by confirmatory sequencing at the Ohio State University facility. The shRNA sequences (of final siRNA products) are listed in Table S2. For lentivirus packaging, lentivector plasmids were transfected into HEK293T cells together with packaging and envelope plasmids (psPAX2 and pMD2.G) using Lipofectamine 3000 (Thermo Fisher Scientific, L3000008). Three days after transfection, the medium was passed through a filter of $0.45 \mu \mathrm{m}$ 
pore size and then used for transduction of ARPE19 cells. After 48 hours of infection, cells were selected with $1 \mu \mathrm{g} / \mathrm{ml}$ of Puromycin (Thermo Fisher Scientific, A1113803) for 5-10 days.

\section{Plasmid and siRNA transfection}

Plasmids were transfected via co-incubation with Lipofectamine 3000 for $24 \mathrm{~h}$ in the recipient cell culture following the manufacturer's instructions. The medium was then replaced with fresh DMEM/F12 for the cells to recover (24h) before their further use in various analyses.

For siRNA transfection, ARPE19 cells were cultured to $80 \%$ confluency in the DMEM/F12 medium containing 10\% FBS in 6-well plates, and then added with a scrambled or BRD2-, BRD4-, or SREBP1-specific siRNA (sequence information available at the manufacturer, Thermo Fisher Scientific). The cells were transfected overnight using the Lipofectamine RNAi Max transfection reagent (Thermo Fisher Scientific, 13778150), and then recovered in the DMEM/F12 culture medium for $24 \mathrm{~h}$ before further experimental use.

\section{Western blotting}

Western blot analysis was performed following our published protocol ${ }^{38}$ with minor modification. Briefly, cells were lysed with the Pierce RIPA lysis buffer (Thermo Fisher Scientific, 89901) containing Halt Protease Inhibitor Cocktail (Thermo Fisher Scientific, 87785). Total protein concentration was determined using the DC Protein Assay kit (Bio-Rad, 5000111). The cell lysates were solubilized in Pierce Lane Marker Non-Reducing Sample Buffer (Thermo Fisher Scientific, 39001) and heated at $95^{\circ} \mathrm{C}$ for $10 \mathrm{~min}$ prior to SDS-PAGE and Western blotting. The information for the antibodies used are available in Table S3. Specific protein bands on Western blots were quantified by the ImageJ 64 software (https://imagej.nih.gov/ij/) using Gel analyzer script. Densitometry data were normalized to loading control (GAPDH or $\beta$-actin) and then to a basal condition (e.g. vehicle and/or scrambled sequence siRNA).

\section{Co-immunoprecipitation (co-IP)}

pEGFP-N1-FLAG (empty vector) (Addgene, 60360) and pFLAG-SREBP2Nt (N-terminal transcriptionally active domain, amino acids 1-482) (Addgene, 26807) were used to transfect cells (HEK293). For co-IP, cells were lysed on ice for 30min in Pierce IP Lysis Buffer (Thermo Fisher Scientific, 87788) containing Halt Protease Inhibitor Cocktail (Thermo Fisher Scientific, $87785)$, and then centrifuged at $12000 \mathrm{rpm}$ for $15 \mathrm{~min}$ at $4^{\circ} \mathrm{C}$. The supernatant was incubated with $50 \mu$ l of Pierce Anti-DYKDDDDK Magnetic Agarose beads (Thermo Fisher Scientific, A36797) at $4^{\circ} \mathrm{C}$ overnight. The beads were washed $3 x$ with cold PBS buffer and then incubated in $0.1 \mathrm{M}$ glycine $(\mathrm{pH} 2.8)$ for $10 \mathrm{~min}$ at room temperature with frequent vortex to elute the immuno-precipitates. The eluate was neutralized with $1 \mathrm{M} \mathrm{Tris-HCl,} \mathrm{pH} 8.5(15 \mu \mathrm{l}$ per $100 \mu \mathrm{l}$ eluate), and briefly heated at $95^{\circ} \mathrm{C}$ prior to its use for SDS-PAGE and Western blot analysis.

\section{Chromatin immunoprecipitation (ChIP) analysis}

ChIP analysis was performed by using the Pierce Magnetic ChIP kit (Thermo Fisher Scientific, 26157) and following the manufacturer's manual. Briefly, formaldehyde (final concentration 1\%) was incubated with the ARPE19 cell culture to cross-link protein with DNA for $10 \mathrm{~min}$, and the reaction was then quenched with a glycine solution for $5 \mathrm{~min}$. Cells were washed with ice-cold PBS, lysed in the Membrane Extraction buffer, and centrifuged at $3000 \mathrm{~g}$ for $5 \mathrm{~min}$ to collect the nuclei. The nuclear pellets were re-suspended in $200 \mu$ of MNase Digestion Buffer Working Solution and then digested by incubation with MNase at $37^{\circ} \mathrm{C}$ for $15 \mathrm{~min}$. The reaction was terminated in MNase Stop Solution. The nuclei were recovered by centrifugation at $9000 \mathrm{~g}$ for 5 
min, re-suspended in IP Dilution Buffer, and then sonicated (four 5-second pulses at 20 Watts for $2 \times 10^{6}$ cells) to break the nuclear membrane. Following centrifugation at $9000 \mathrm{~g}$ for $5 \mathrm{~min}$, the supernatant was collected and incubated overnight with a BRD2 antibody (Table S3) or IgG control (5 $\mu \mathrm{g}$ antibody per reaction). ChIP-grade Protein A/G Magnetic beads were added and incubated overnight at $4^{\circ} \mathrm{C}$ on mixing. The beads were collected and washed sequentially with IP Wash Buffer-1, IP Wash Buffer-2 and then resuspended in the elution buffer. The proteinDNA cross-link was reversed with $5 \mathrm{M} \mathrm{NaCl}$ followed by RNA and protein digestion with RNAse $A$ and Proteinase K. The DNA pulldown was purified with DNA Clean-Up Column and used for qPCR. Primers used for detection of S2R and SREBP promoter regions that contain predicted SREBP-binding sterol-response consensus elements are listed in Table S4. For each qPCR assay, triplicate samples were used, and data were normalized to respective input samples. For BRD2 gain of function, we selected a BRD2-expressing stable ARPE19 cell line using a lentivector constructed based on an empty vector (Addgene, 19319).

\section{Statistical analysis}

Repeat experiments were performed on different $(n \geq 3)$ occasions. Results were plotted as mean \pm SEM unless otherwise specified. Statistical significance (set at $P<0.05$ ) was determined by one-way ANOVA with Bonferroni post-hoc test for multi-group comparison or two-tailed paired Student's t test for two-group comparison (GraphPad Prism 7). Significance is indicated as $* \mathrm{P}<0.05, * * * \mathrm{p}<0.01$, or $* * * \mathrm{P}<0.001$; no significance is labeled as " $\mathrm{ns}$ " or not labeled, as specified in each figure legend. 


\section{References}

1. Chu BB, Liao YC, Qi W, Xie C, Du X, Wang J, Yang H, Miao HH, Li BL and Song BL. Cholesterol transport through lysosome-peroxisome membrane contacts. Cell. 2015;161:291-306.

2. Ebrahimi-Fakhari D, Wahlster L, Bartz F, Werenbeck-Ueding J, Praggastis M, Zhang J, Joggerst-Thomalla B, Theiss S, Grimm D, Ory DS and Runz H. Reduction of TMEM97 increases NPC1 protein levels and restores cholesterol trafficking in Niemann-pick type C1 disease cells. Hum Mol Genet. 2016;25:3588-3599.

3. Bartz F, Kern L, Erz D, Zhu M, Gilbert D, Meinhof T, Wirkner U, Erfle H, Muckenthaler M, Pepperkok $\mathrm{R}$ and Runz $\mathrm{H}$. Identification of cholesterol-regulating genes by targeted RNAi screening. Cell Metab. 2009;10:63-75.

4. Alon A, Schmidt HR, Wood MD, Sahn JJ, Martin SF and Kruse AC. Identification of the gene that codes for the sigma2 receptor. Proc Natl Acad Sci U S A. 2017.

5. Sanchez-Pulido $L$ and Ponting CP. TM6SF2 and MAC30, new enzyme homologs in sterol metabolism and common metabolic disease. Front Genet. 2014;5:439.

6. Matsumoto RR, Nguyen L, Kaushal N and Robson MJ. Sigma (sigma) receptors as potential therapeutic targets to mitigate psychostimulant effects. Adv Pharmacol. 2014;69:323-86.

7. Zeng $\mathrm{C}$, McDonald ES and Mach $\mathrm{RH}$. Molecular Probes for Imaging the Sigma-2 Receptor: In Vitro and In Vivo Imaging Studies. Handb Exp Pharmacol. 2017;244:309-330.

8. Bradner JE, Hnisz D and Young RA. Transcriptional Addiction in Cancer. Cell. 2017; 168:629-643.

9. Shi J and Vakoc CR. The mechanisms behind the therapeutic activity of BET bromodomain inhibition. Molecular cell. 2014;54:728-36.

10. Brown JD, Feldman ZB, Doherty SP, Reyes JM, Rahl PB, Lin CY, Sheng Q, Duan Q, Federation AJ, Kung AL, Haldar SM, Young RA, Plutzky J and Bradner JE. BET bromodomain proteins regulate enhancer function during adipogenesis. Proceedings of the National Academy of Sciences of the United States of America. 2018;115:2144-2149.

11. Sakamaki JI, Wilkinson S, Hahn M, Tasdemir N, O'Prey J, Clark W, Hedley A, Nixon C, Long JS, New M, Van Acker T, Tooze SA, Lowe SW, Dikic I and Ryan KM. Bromodomain Protein BRD4 Is a Transcriptional Repressor of Autophagy and Lysosomal Function. Molecular cell. 2017;66:517-532 e9.

12. Andrieu GP, Shafran JS, Deeney JT, Bharadwaj KR, Rangarajan A and Denis GV. BET proteins in abnormal metabolism, inflammation, and the breast cancer microenvironment. $J$ Leukoc Biol. 2018;104:265-274.

13. Shin DG and Bayarsaihan D. A Novel Epi-drug Therapy Based on the Suppression of BET Family Epigenetic Readers. Yale J Biol Med. 2017;90:63-71.

14. Curcio CA. Soft Drusen in Age-Related Macular Degeneration: Biology and Targeting Via the Oil Spill Strategies. Invest Ophthalmol Vis Sci. 2018;59:AMD160-AMD181.

15. Filippakopoulos $P$ and Knapp $S$. Targeting bromodomains: epigenetic readers of lysine acetylation. Nature reviews Drug discovery. 2014;13:337-56.

16. Filippakopoulos P, Picaud S, Mangos M, Keates T, Lambert JP, Barsyte-Lovejoy D, Felletar I, Volkmer R, Muller S, Pawson T, Gingras AC, Arrowsmith CH and Knapp S. Histone recognition and large-scale structural analysis of the human bromodomain family. Cell. 2012;149:214-31.

17. Wang CY and Filippakopoulos P. Beating the odds: BETs in disease. Trends in biochemical sciences. 2015;40:468-79.

18. Ramalho-Carvalho J, Goncalves CS, Graca I, Bidarra D, Pereira-Silva E, Salta S, Godinho MI, Gomez A, Esteller M, Costa BM, Henrique R and Jeronimo C. A multiplatform 
approach identifies miR-152-3p as a common epigenetically regulated onco-suppressor in prostate cancer targeting TMEM97. Clin Epigenetics. 2018;10:40.

19. Sahn JJ, Mejia GL, Ray PR, Martin SF and Price TJ. Sigma 2 Receptor/Tmem97 Agonists Produce Long Lasting Antineuropathic Pain Effects in Mice. ACS Chem Neurosci. 2017;8:1801-1811.

20. Mondal S, Hegarty E, Sahn JJ, Scott LL, Gokce SK, Martin C, Ghorashian N, Satarasinghe PN, lyer S, Sae-Lee W, Hodges TR, Pierce JT, Martin SF and Ben-Yakar A. High-Content Microfluidic Screening Platform Used To Identify sigma2R/Tmem97 Binding Ligands that Reduce Age-Dependent Neurodegeneration in C. elegans SC_APP Model. ACS Chem Neurosci. 2018;9:1014-1026.

21. Scott LL, Sahn JJ, Ferragud A, Yen RC, Satarasinghe PN, Wood MD, Hodges TR, Shi T, Prakash BA, Friese KM, Shen A, Sabino V, Pierce JT and Martin SF. Small molecule modulators of sigma2R/Tmem97 reduce alcohol withdrawal-induced behaviors.

Neuropsychopharmacology. 2018;43:1867-1875.

22. Nicholson H, Comeau A, Mesangeau C, McCurdy CR and Bowen WD. Characterization of CM572, a Selective Irreversible Partial Agonist of the Sigma-2 Receptor with Antitumor Activity. J Pharmacol Exp Ther. 2015;354:203-12.

23. Schmit $\mathrm{K}$ and Michiels C. TMEM Proteins in Cancer: A Review. Front Pharmacol. 2018;9:1345.

24. Xu Y and Vakoc CR. Targeting Cancer Cells with BET Bromodomain Inhibitors. Cold Spring Harb Perspect Med. 2017;7.

25. Devaiah BN, Gegonne A and Singer DS. Bromodomain 4: a cellular Swiss army knife. J Leukoc Biol. 2016;100:679-686.

26. Devaiah BN, Lewis BA, Cherman N, Hewitt MC, Albrecht BK, Robey PG, Ozato K, Sims RJ, 3rd and Singer DS. BRD4 is an atypical kinase that phosphorylates serine2 of the RNA polymerase II carboxy-terminal domain. Proceedings of the National Academy of Sciences of the United States of America. 2012;109:6927-32.

27. Devaiah BN, Case-Borden C, Gegonne A, Hsu CH, Chen Q, Meerzaman D, Dey A, Ozato $\mathrm{K}$ and Singer DS. BRD4 is a histone acetyltransferase that evicts nucleosomes from chromatin. Nature structural \& molecular biology. 2016.

28. Nicodeme E, Jeffrey KL, Schaefer U, Beinke S, Dewell S, Chung CW, Chandwani R, Marazzi I, Wilson P, Coste H, White J, Kirilovsky J, Rice CM, Lora JM, Prinjha RK, Lee K and Tarakhovsky A. Suppression of inflammation by a synthetic histone mimic. Nature. 2010;468:1119-23.

29. Delmore JE, Issa GC, Lemieux ME, Rahl PB, Shi J, Jacobs HM, Kastritis E, Gilpatrick T, Paranal RM, Qi J, Chesi M, Schinzel AC, McKeown MR, Heffernan TP, Vakoc CR, Bergsagel PL, Ghobrial IM, Richardson PG, Young RA, Hahn WC, Anderson KC, Kung AL, Bradner JE and Mitsiades CS. BET bromodomain inhibition as a therapeutic strategy to target c-Myc. Cell. 2011;146:904-17.

30. Lee JE, Park YK, Park S, Jang Y, Waring N, Dey A, Ozato K, Lai B, Peng W and Ge K. Brd4 binds to active enhancers to control cell identity gene induction in adipogenesis and myogenesis. Nature communications. 2017;8:2217.

31. Sabari BR, Dall'Agnese A, Boija A, Klein IA, Coffey EL, Shrinivas K, Abraham BJ, Hannett NM, Zamudio AV, Manteiga JC, Li CH, Guo YE, Day DS, Schuijers J, Vasile E, Malik S, Hnisz D, Lee TI, Cisse, II, Roeder RG, Sharp PA, Chakraborty AK and Young RA. Coactivator condensation at super-enhancers links phase separation and gene control. Science. 2018;361.

32. Segatto M, Fittipaldi R, Pin F, Sartori R, Dae Ko K, Zare H, Fenizia C, Zanchettin G, Pierobon ES, Hatakeyama S, Sperti C, Merigliano S, Sandri M, Filippakopoulos P, Costelli $P$, Sartorelli V and Caretti G. Epigenetic targeting of bromodomain protein BRD4 counteracts cancer cachexia and prolongs survival. Nature communications. 2017;8:1707. 
33. Bailey D, Jahagirdar R, Gordon A, Hafiane A, Campbell S, Chatur S, Wagner GS, Hansen HC, Chiacchia FS, Johansson J, Krimbou L, Wong NC and Genest J. RVX-208: a small molecule that increases apolipoprotein A-I and high-density lipoprotein cholesterol in vitro and in vivo. Journal of the American College of Cardiology. 2010;55:2580-9.

34. Brown JD, Lin CY, Duan Q, Griffin G, Federation AJ, Paranal RM, Bair S, Newton G, Lichtman AH, Kung AL, Yang T, Wang H, Luscinskas FW, Croce KJ, Bradner JE and Plutzky J. NF-kappaB directs dynamic super enhancer formation in inflammation and atherogenesis. Molecular cell. 2014;56:219-31.

35. Whyte WA, Orlando DA, Hnisz D, Abraham BJ, Lin CY, Kagey MH, Rahl PB, Lee TI and Young RA. Master transcription factors and mediator establish super-enhancers at key cell identity genes. Cell. 2013;153:307-19.

36. Vazquez-Rosa E, Watson MR, Sahn JJ, Hodges TR, Schroeder RE, Cintron-Perez CJ, Shin MK, Yin TC, Emery JL, Martin SF, Liebl DJ and Pieper A. Neuroprotective Efficacy of a Novel Sigma 2 Receptor/TMEM97 Modulator (DKR-1677) after Traumatic Brain Injury. ACS Chem Neurosci. 2018.

37. Tesei A, Cortesi M, Zamagni A, Arienti C, Pignatta S, Zanoni M, Paolillo M, Curti D, Rui M, Rossi D and Collina S. Sigma Receptors as Endoplasmic Reticulum Stress "Gatekeepers" and their Modulators as Emerging New Weapons in the Fight Against Cancer. Front Pharmacol. 2018;9:711.

38. Wang B, Zhang M, Takayama T, Shi X, Roenneburg DA, Kent KC and Guo LW. BET Bromodomain Blockade Mitigates Intimal Hyperplasia in Rat Carotid Arteries. EBioMedicine. 2015;2:1650-61. 


\section{Figure legends}

\section{Figure 1. Pan-BETs inhibition prevents S2R upregulation upon cytosolic cholesterol deprivation}

ARPE19 cells were cultured to an $~ 70-80 \%$ confluency in the DMEM/F12 medium containing $10 \%$ FBS. The cells were incubated with U188866A (abbreviated as U18, final $5 \mu \mathrm{M}$ ) for $24 \mathrm{~h}$ before qRT-PCR (A and B) assay, Western blotting (C and D), or filipin staining of cholesterol (E). For starvation treatment, the medium was changed to that containing $0 \% \mathrm{FBS}$. For pretreatment to inhibit BETs, JQ1 $(1 \mu \mathrm{M})$ or vehicle control (equal amount of DMSO) was included in the cell culture during the treatment with U18 or starvation.

Quantification: At least three independent repeat experiments were performed; data were normalized to GAPDH (qRT-PCR) or $\beta$-actin (Western blot) and then to the control of vehicle only (no U18, no starvation). The normalized data were averaged $(n \geq 3)$ to calculate mean \pm SEM. Statistics: One-way ANOVA with Bonferroni post-hoc test; ${ }^{*} \mathrm{P}<0.05$, ${ }^{* \star *} \mathrm{P}<0.001$; ns, no significance.

\section{Figure 2. Inhibitory effect of BRD2 silencing on S2R mRNA and protein expression}

ARPE19 cells were transfected with a BRD2-specific siRNA or infected with lentivirus to express a BRD3- or BRD4-specific shRNA prior to a 24-h U18 or starvation treatment, as described in detail in Methods. Cells were then harvested for assays.

A-C. qRT-PCR. Shown is each representative of two similar yet independent experiments. Mean $\pm S D, n=3$.

D-F. Western blot. Data were quantified as described in the Figure 1 legend. Mean $\pm S E M, n=4$ independent repeat experiments.

Statistics (A-F): One-way ANOVA with Bonferroni post-hoc test; ${ }^{*} \mathrm{P}<0.05,{ }^{* *} \mathrm{P}<0.01,{ }^{* * *} \mathrm{P}<0.001$, compared between conditions represented by light- and dark-colored bars (e.g. scrambled and BRD2 siRNA represented by light red and dark red in A, respectively); for simplicity, nonsignificant comparison is not labeled.

\section{Figure 3. Effect of BRD2/BRD4 double silencing on S2R protein levels}

Experiments were performed as described for Figure 2 except that BRD2 and BRD4 were silenced either separately with their specific siRNAs or simultaneously with combined siRNAs. $A$ (BETs) and B (S2R) were from the same set of experiments, and so were $C / D$ and $E / F$. Data were quantified as described in the Figure 1 legend. Statistics: One-way ANOVA with Bonferroni post-hoc test; $n=4$ independent repeat experiments; ${ }^{*} P<0.05,{ }^{* \star} P<0.01,{ }^{* *} P<0.001$, compared between conditions represented by a pair of light- and dark-colored bars (e.g. grey and black: scrambled and BRD-specific siRNA); non-significant comparison is not labeled.

\section{Figure 4. Effect of SREBP2 or SREBP1 silencing on S2R expression}

Experiments were performed as described for Figure 2 except that SREBP2 or SREBP1 silencing was induced. A and B. qRT-PCR. Each represents one of two similar experiments. C and D. Western blot. Data were quantified as described in the Figure 1 legend. Statistics: One- 
way ANOVA with Bonferroni post-hoc test; $n=4$ independent repeat experiments ( $C$ and $D)$; ${ }^{*} \mathrm{P}<0.05,{ }^{* *} \mathrm{P}<0.01,{ }^{* *} \mathrm{P}<0.001$, compared between conditions represented by a pair of lightand dark-colored bars (e.g. red and dark red); for simplicity, non-significant comparison is not labeled.

\section{Figure 5. Pan-BETs inhibition suppresses the transcription of SREBPs}

Experimental procedures and data quantification methods were the same as that described for Figure 1 (different sets of experiments) except that the main readouts were SREBP2 and SREBP1 mRNA (A, B, E, F, I, J, K) and protein (C, D, G, H) levels.

Statistics: One-way ANOVA with Bonferroni post-hoc test; $n=3$ (qRT-PCR) or 4 (Western blot) independent repeat experiments; ${ }^{\star} \mathrm{P}<0.05$, ${ }^{* *} \mathrm{P}<0.01$, ${ }^{* *} \mathrm{P}<0.001$; for simplicity, non-significant comparison is not labeled.

\section{Figure 6. Effect of BRD2 or BRD4 silencing on SREBP2 or SREBP1 protein levels}

Experimental procedures and data quantification methods were the same as described for Figure 2 (different sets of experiments), except that SREBP2 and SREBP1 protein levels were assessed as a result of BRD2 or BRD4 silencing.

Statistics: One-way ANOVA with Bonferroni post-hoc test; $n=4$ independent repeat experiments; for simplicity, non-significant difference (between a pair of light and dark colors) is not labeled.

\section{Figure 7. BRD2 co-immunoprecipitates with the SREBP2 N-terminal half molecule and S2R gene promoter regions}

Co-immuoprecipitation (A) and ChIP-qPCR (B) experiments were performed as described in detail in Methods. A. Co-immuoprecipitation. Cells were transfected with a vector expressing FLAG-GFP (empty vector control) or FLAG-SREBP2Nt (N-terminal transcriptionally active). IP was performed with an anti-FLAG antibody. Presented blots represent one of three similar experiments (see Figure S3). Statistics: two-tailed paired Student t-test; ${ }^{\star * *} \mathrm{P}<0.001 ; n=3$ independent repeat experiments. B. ChIP-qPCR. Cells were transfected with an empty vector or BRD2-expressing vector. ChIP was performed using an anti-BRD2 antibody. Presented is one of three similar experiments to detect different SREBP-binding regions (software-predicted) of the S2R gene promoter (see Figure S4). Mean $\pm S D ; n=3$ repeats. Statistics: One-way ANOVA with Bonferroni post-hoc test; ${ }^{* \star} P<0.001$. 


\section{Figure 1}
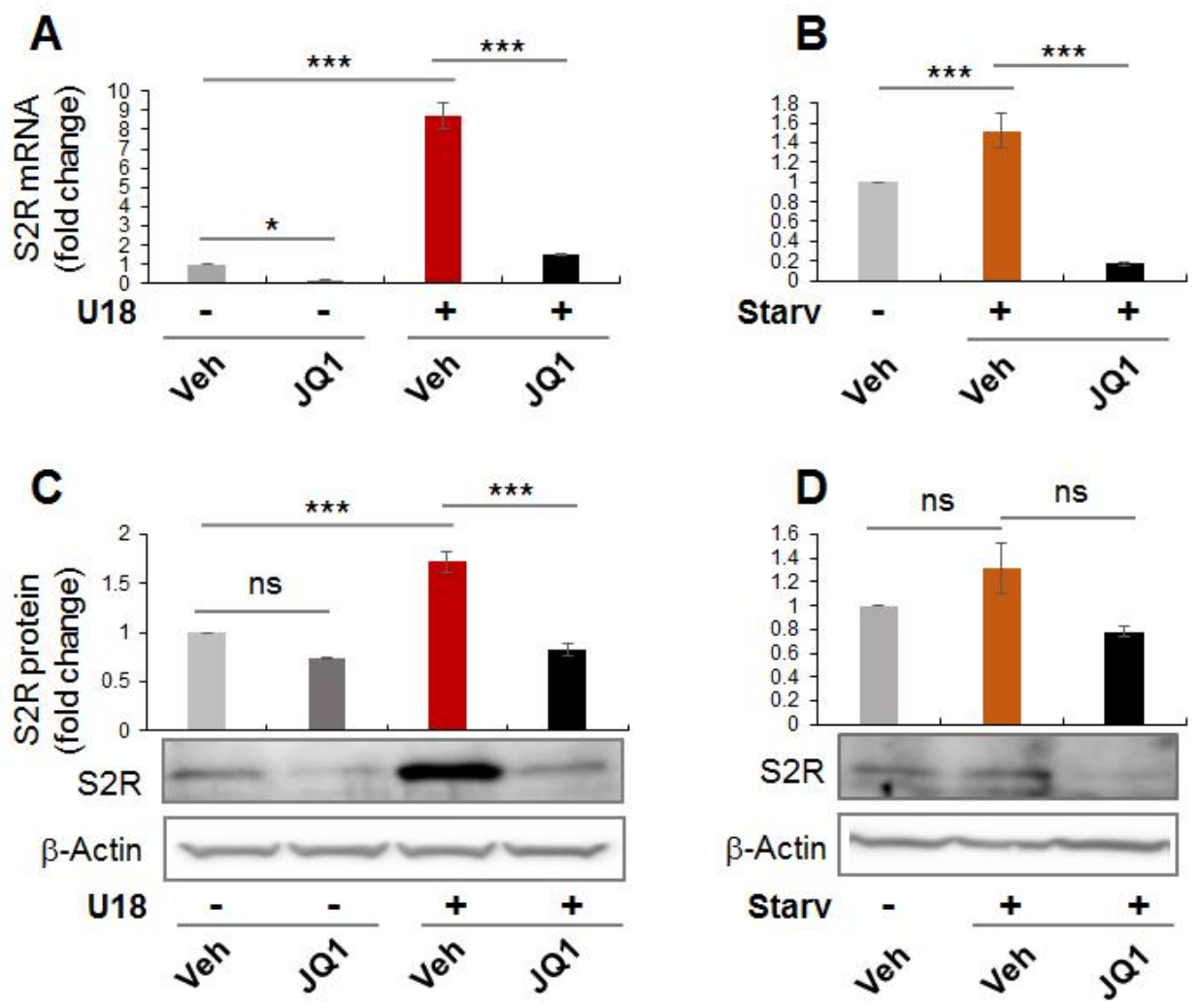

E
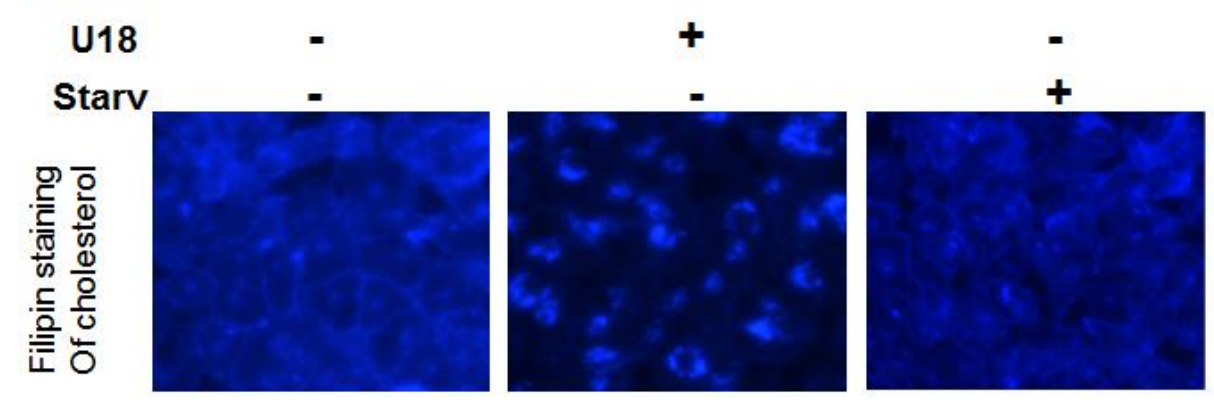
Figure 2
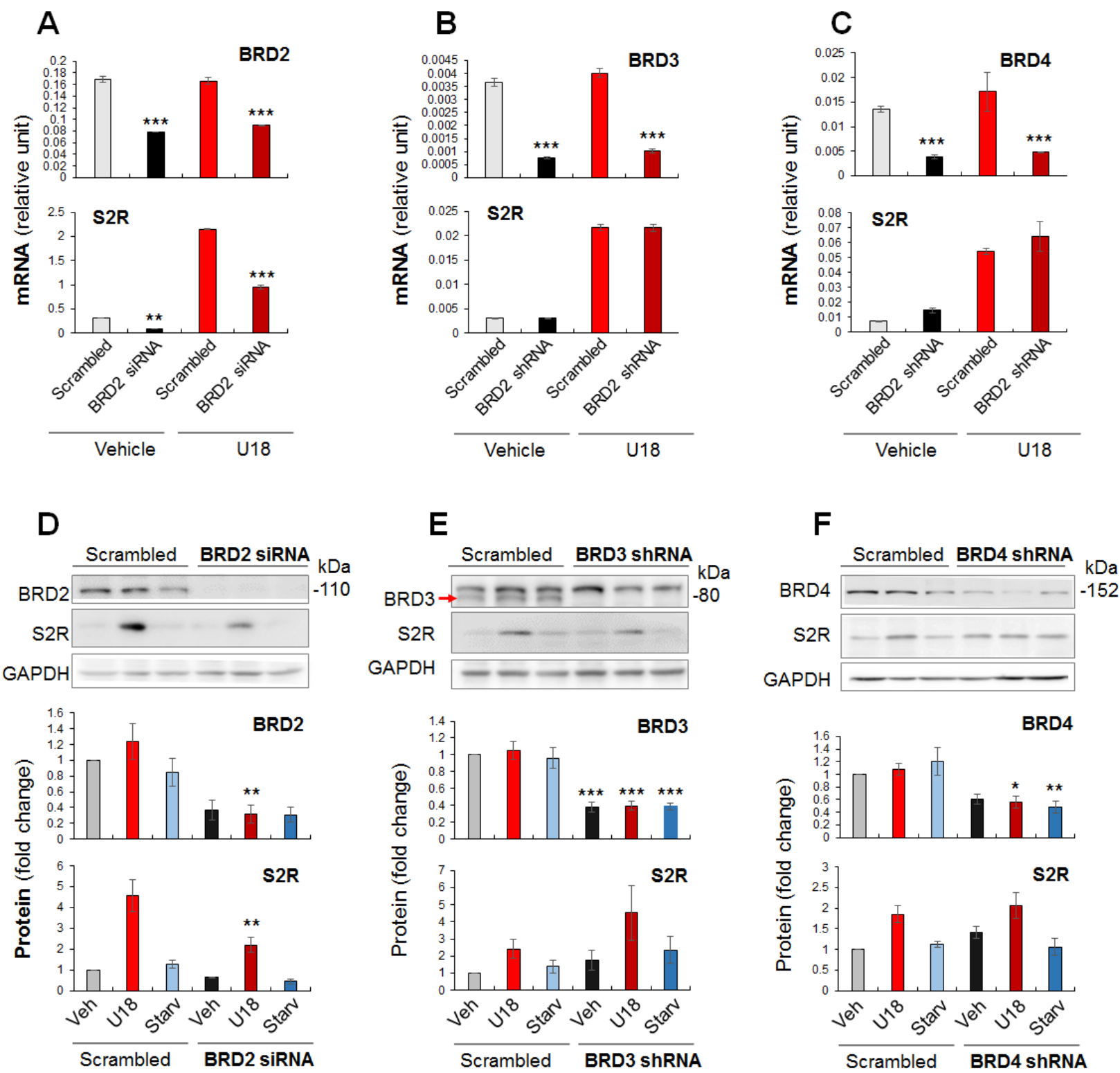
Figure 3
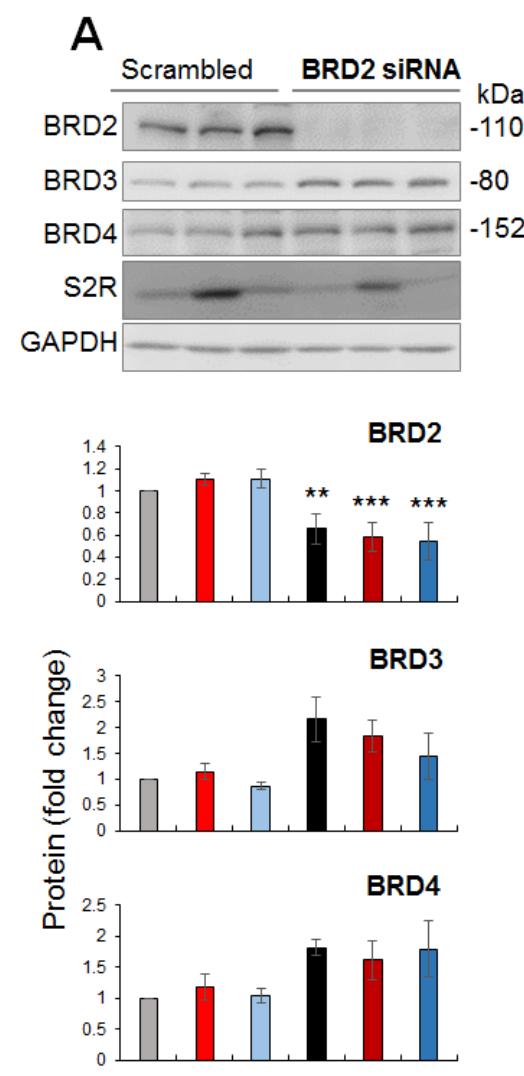

B

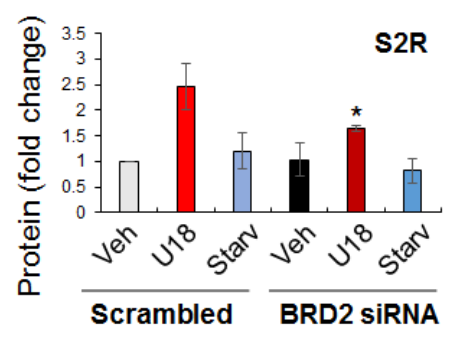

C
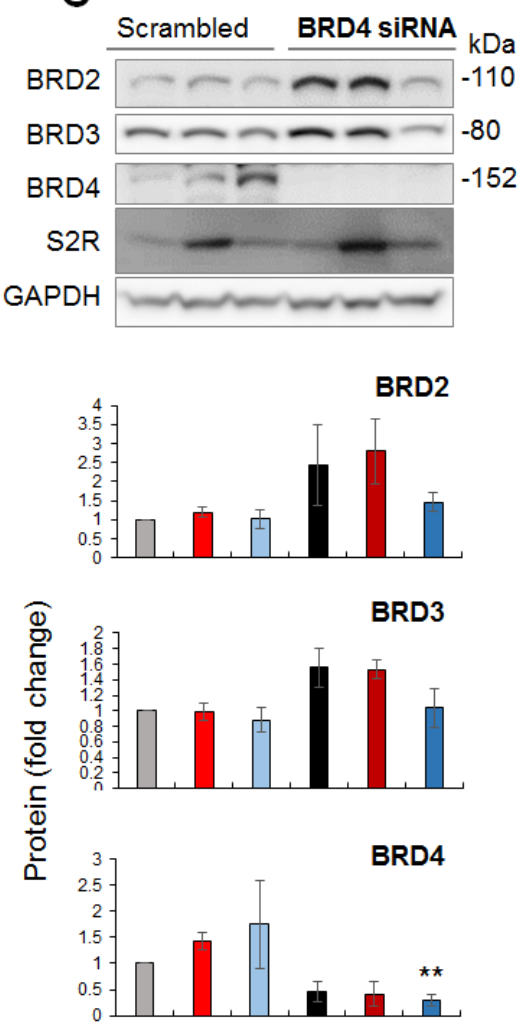

D

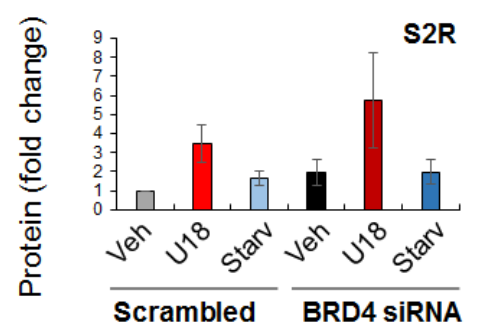

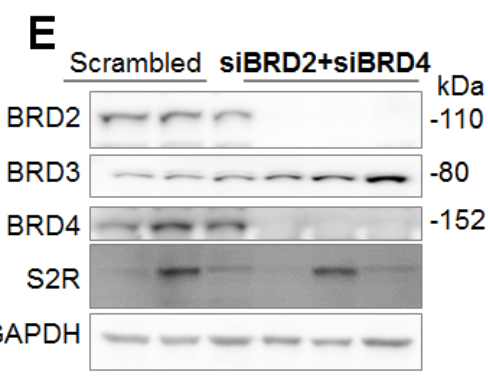

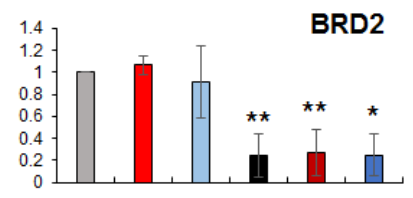

BRD3

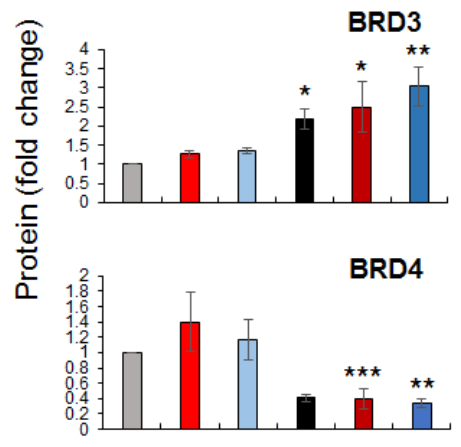

$F$

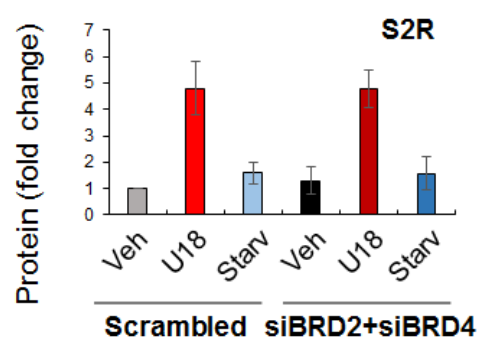


Figure 4
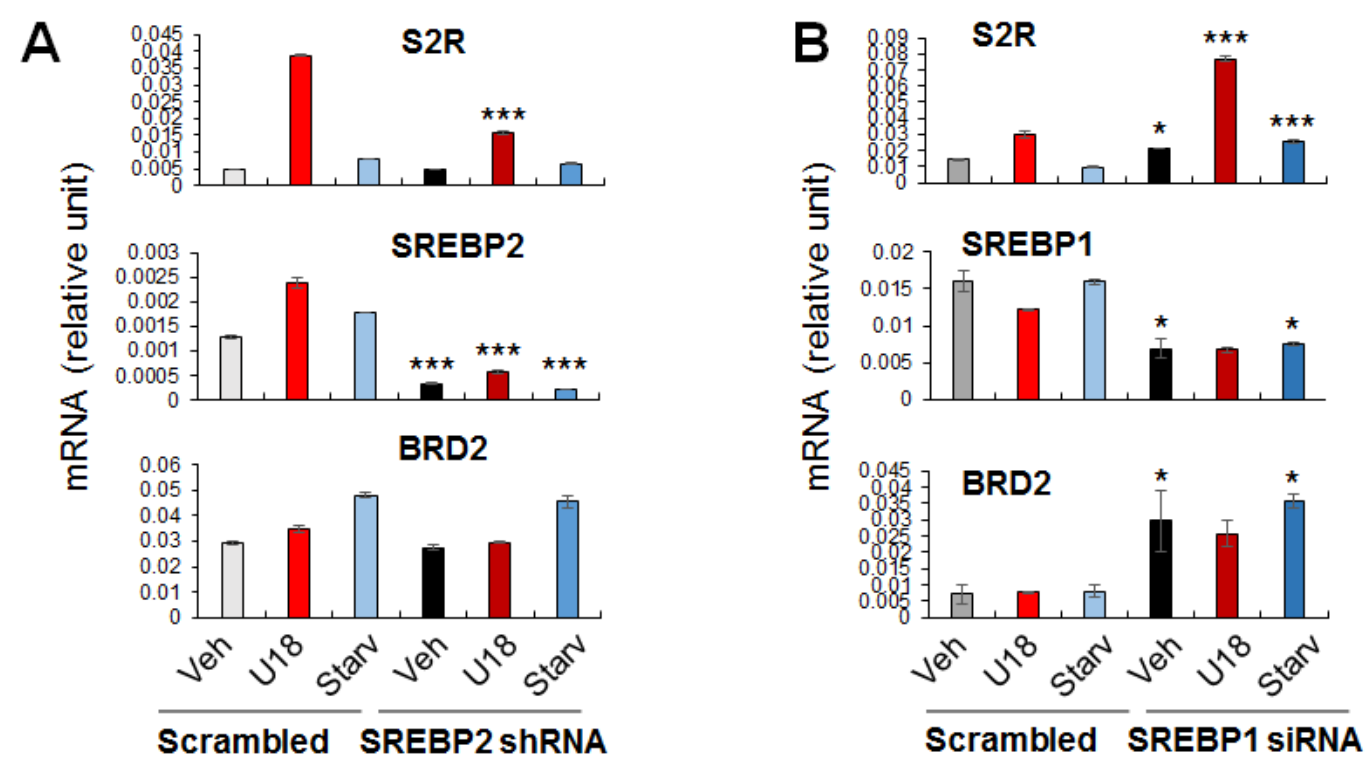

C
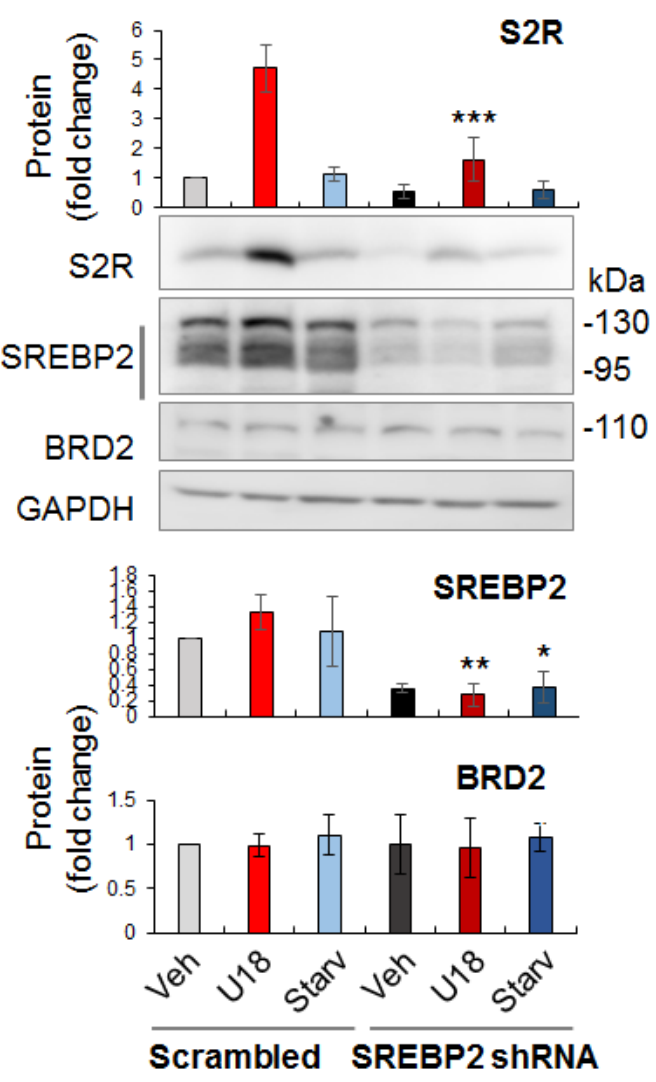

D
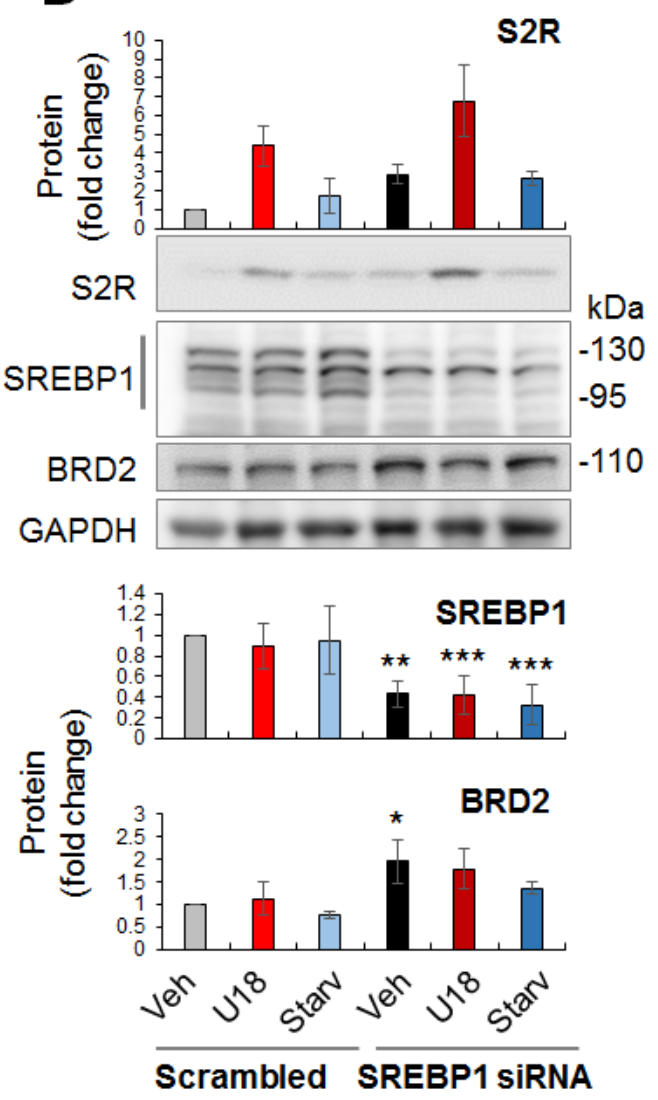
Figure 5
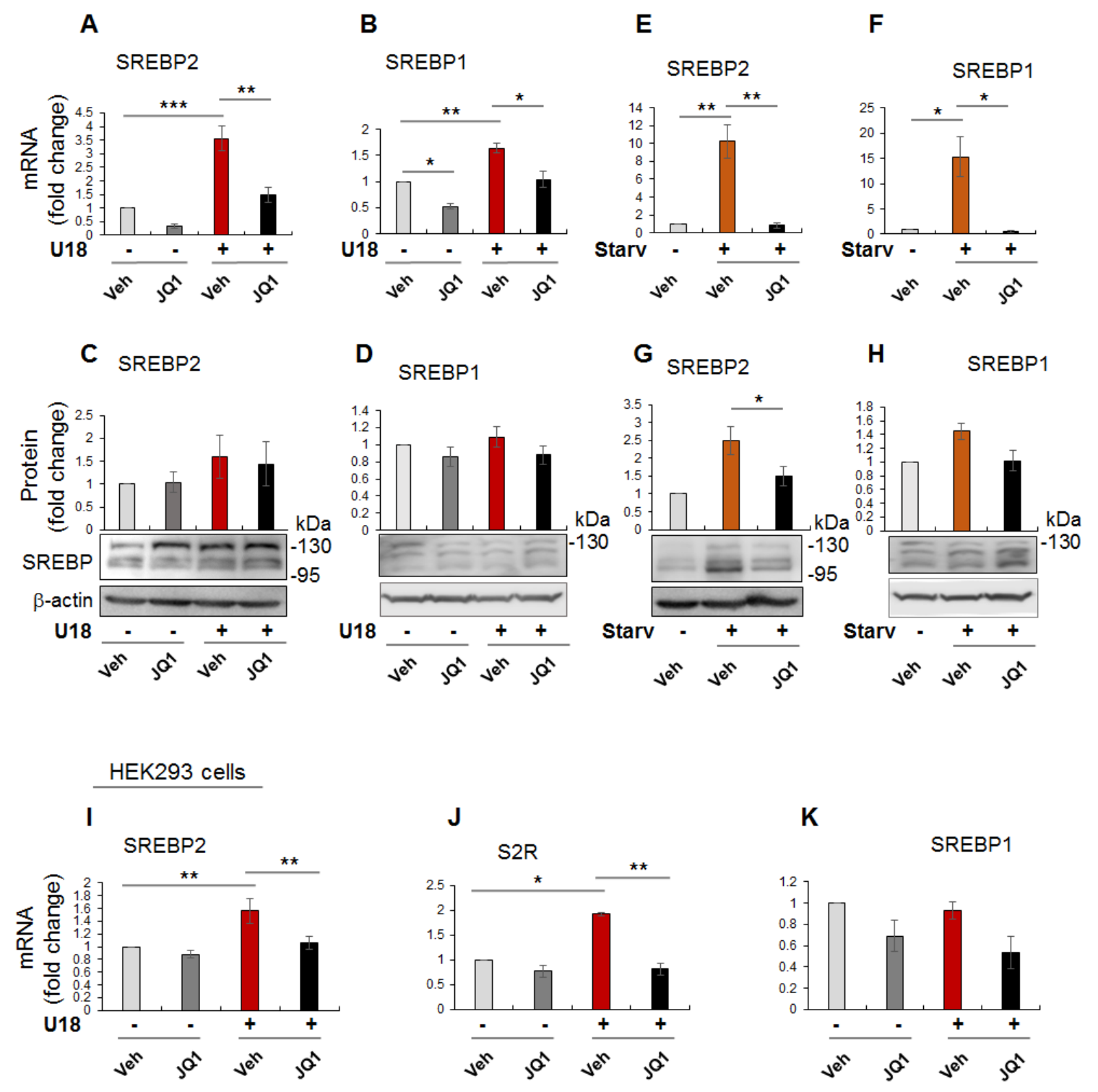


\section{Figure 6}
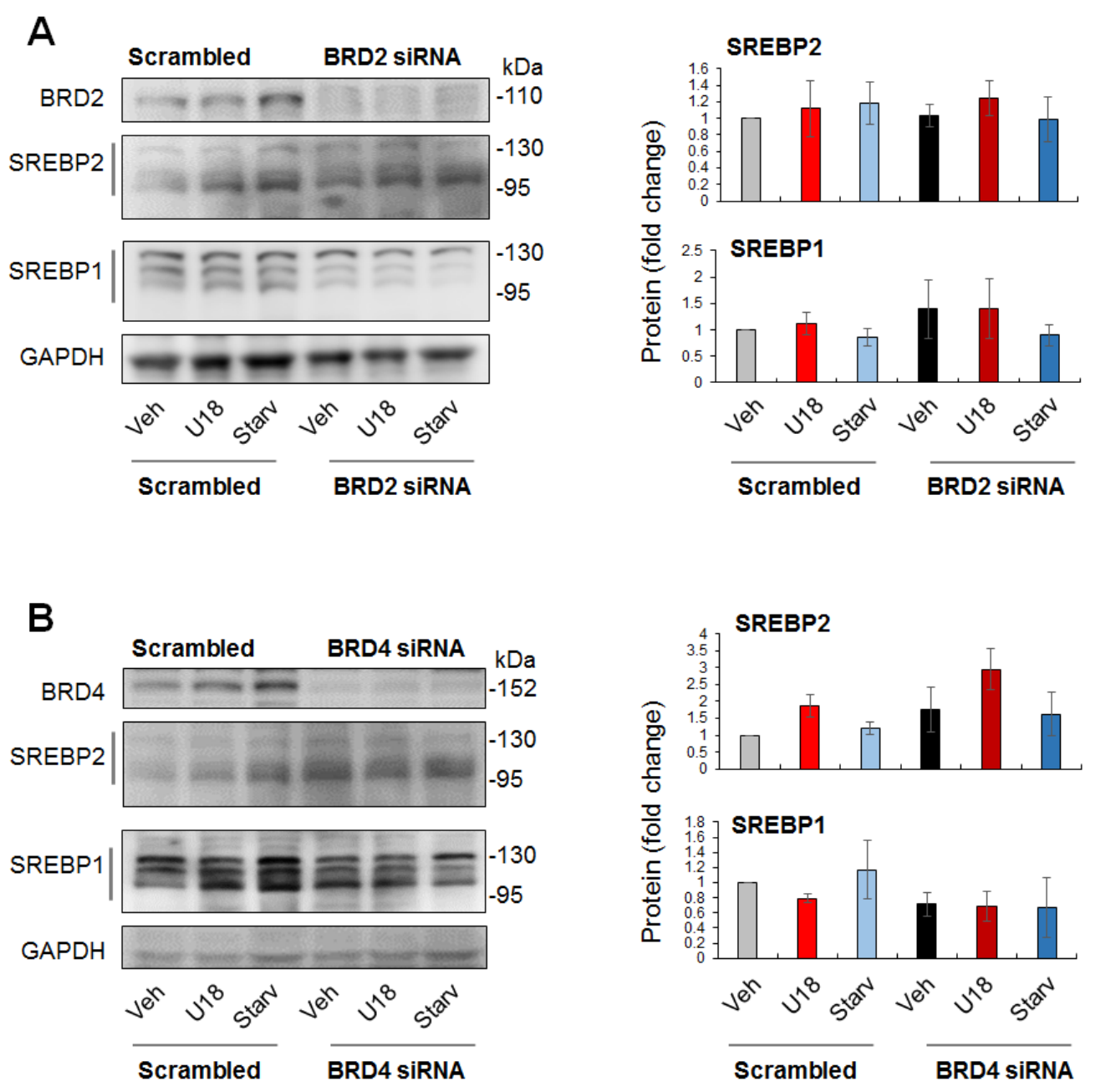
Figure 7

A Co-IP

$\square$ FLAG-GFP

IP: FLAG

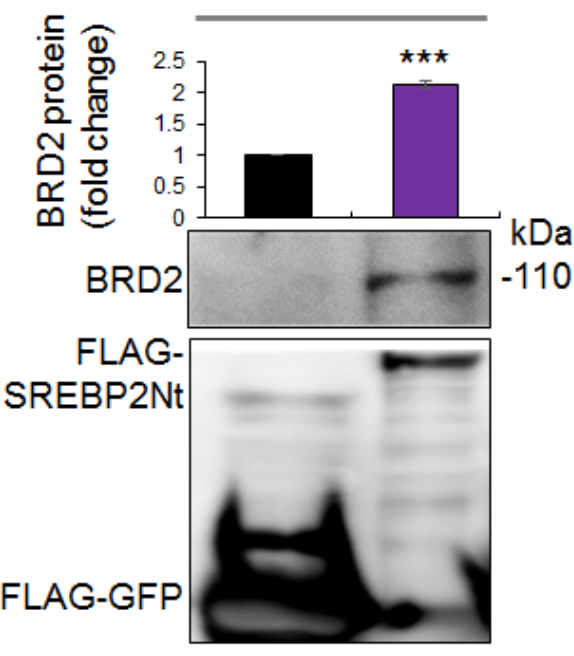

Input(1/10)

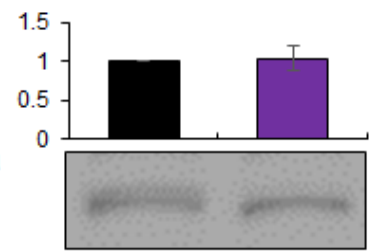

IB: BRD2

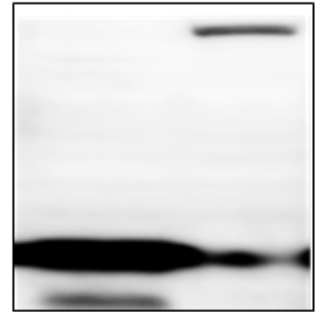

IB: FLAG

\section{B ChIP-qPCR}

IgG $\square$ Anti-BRD2

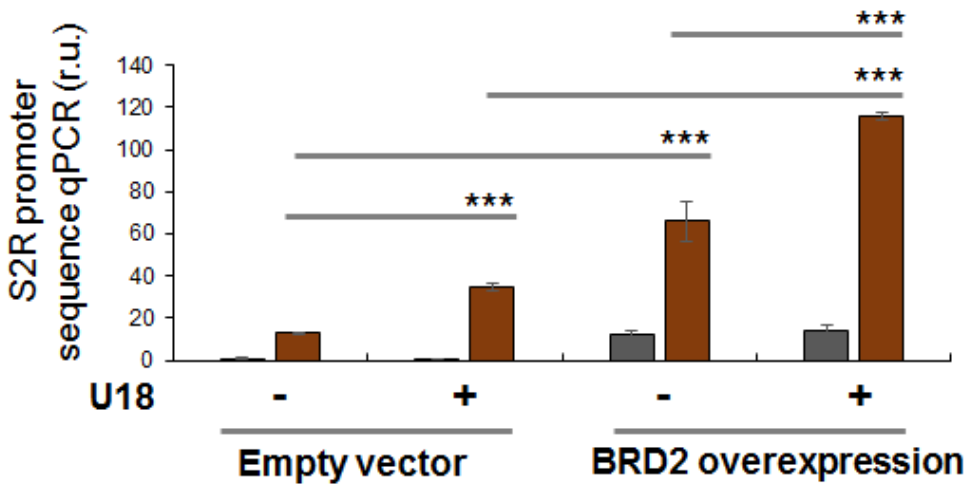


A

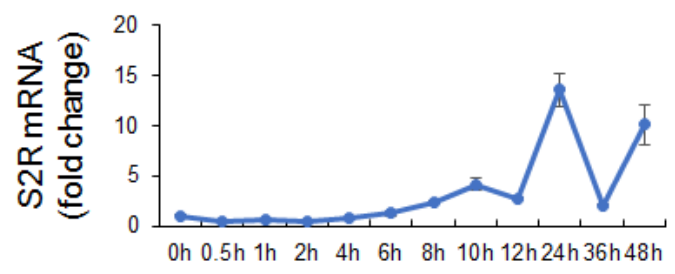

C

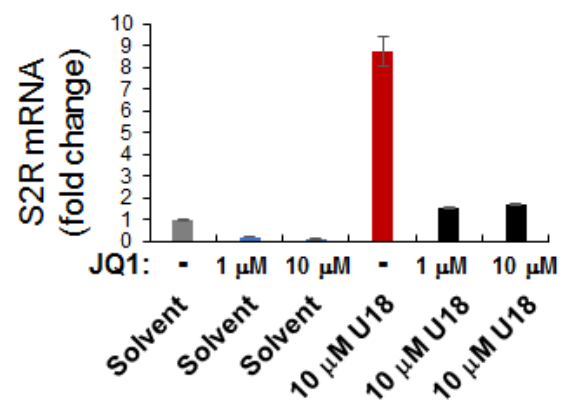

B

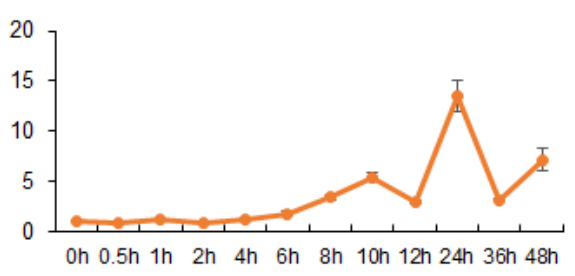

D

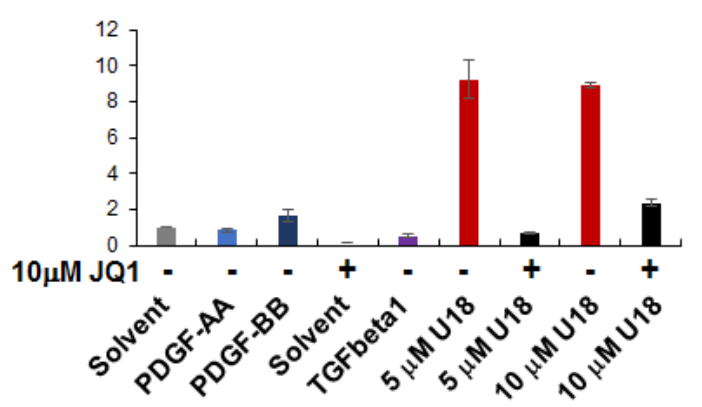

\section{Figure S1. U18 induces upregulation of S2R mRNA in ARPE19 cells}

Experiments were performed as described in Figure 1 except for the various conditions indicated in this figure. For each data point or bar, triplicate samples were used.

A and B. Time course of U18 treatment of ARPE19 cells; two repeat experiments. The data indicates that S2R mRNA levels were maximally increased by U18 $(5 \mu \mathrm{M})$ at $24 \mathrm{~h}$ after treatment.

$\mathrm{C}$ and D. Effects of cytokines and different concentrations of U18 and JQ1 on S2R mRNA expression. PDGF-AA, $20 \mathrm{ng} / \mathrm{ml}$; PDGF-BB, $20 \mathrm{ng} / \mathrm{ml}$; TGF $\beta 1,20 \mathrm{ng} / \mathrm{ml}$. PDGF-AA (cat\#1055AA050) and PDGF-BB (cat\#520BB050) were from R\&D Systems. Human recombinant TGF $\beta 1$ was from Thermo Fisher Scientific (cat\#PHG9214). The data indicate that treatments with $5 \mu \mathrm{M}$ and $10 \mu \mathrm{M}$ U18 were equally effective in inducing S2R mRNA upregulation, and $1 \mu \mathrm{M} \mathrm{JQ1}$ was as potent as $10 \mu \mathrm{M}$ in blocking U18-induced S2R upregulation. 

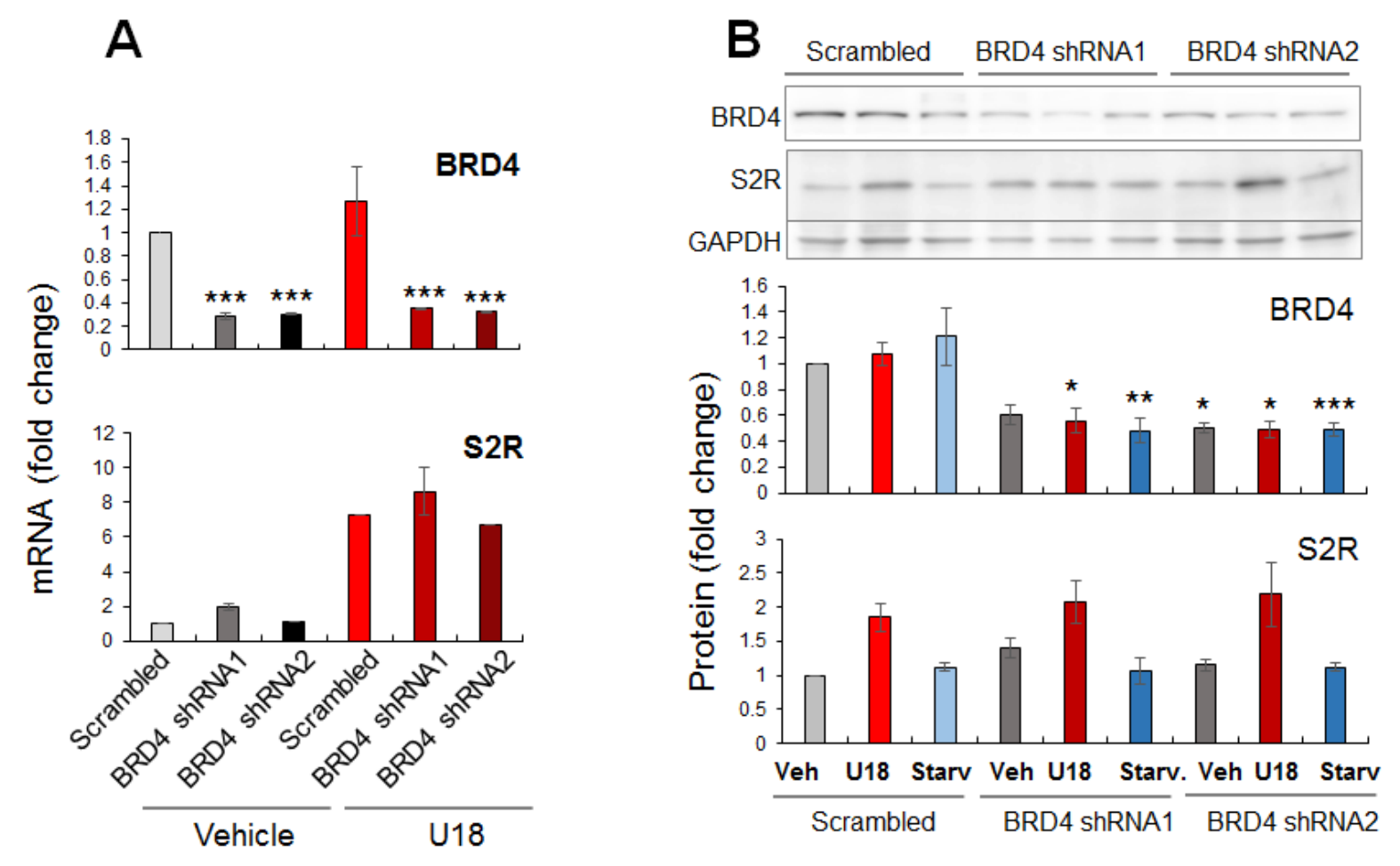

\section{Figure S2. BRD4 silencing does not reduce S2R protein expression}

Experiments were performed as described for Figure 2. Data presented here was obtained from the qRT-PCR $(A)$ and Western blot $(B)$ experiments using two shRNA sequences. The data indicates that experiments using both shRNAs led to similar results. Please refer to corresponding Figure $2 \mathrm{C}$ and $2 \mathrm{~F}$. 


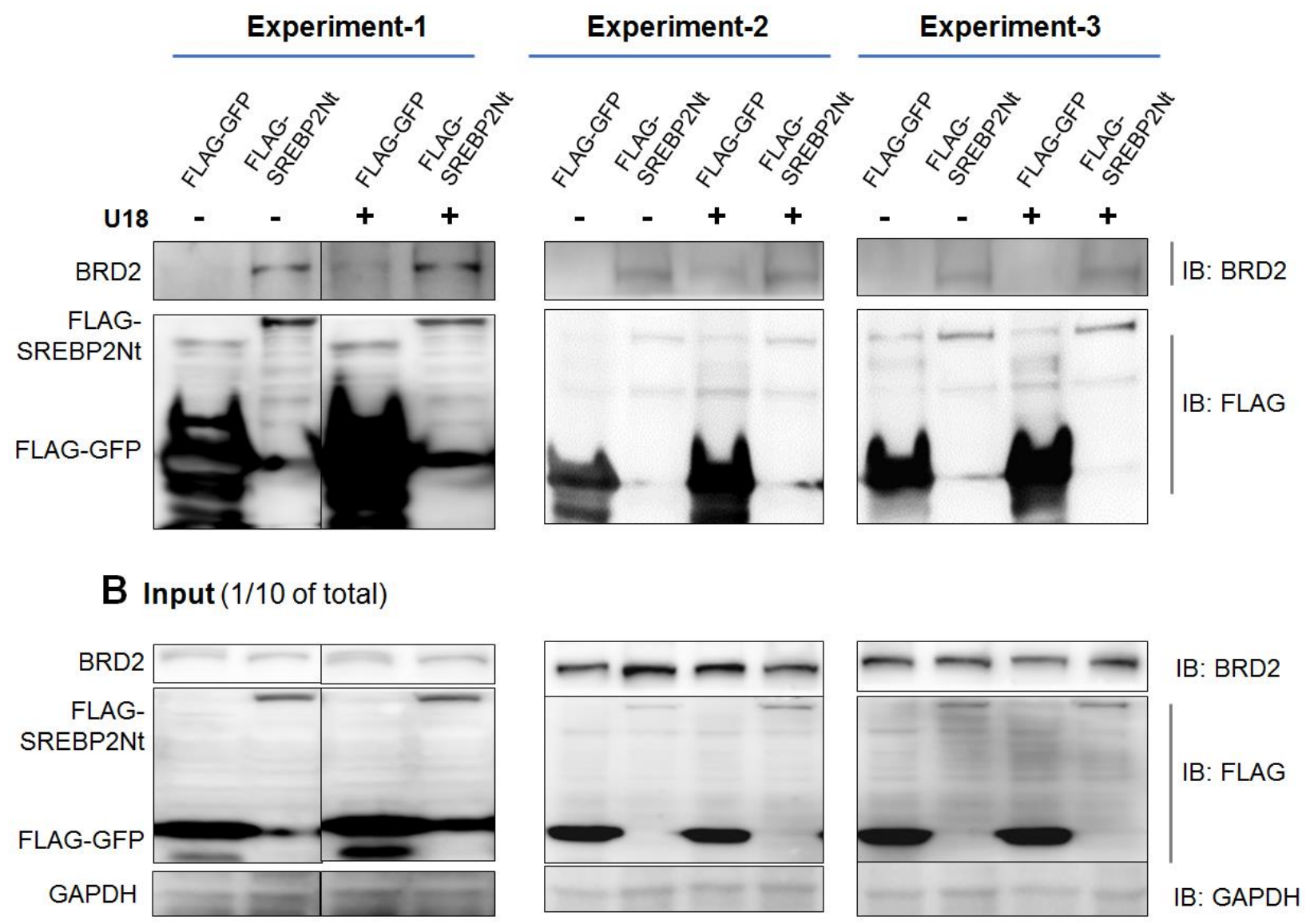

\section{Figure S3. BRD2 co-IPs with the SREBP2 N-terminal TF half molecule}

Experiments were performed as described in Figure 7A. Presented here are the full data from all three repeat experiments that produced the average values and bar graph in Figure $7 \mathrm{~A}$. 


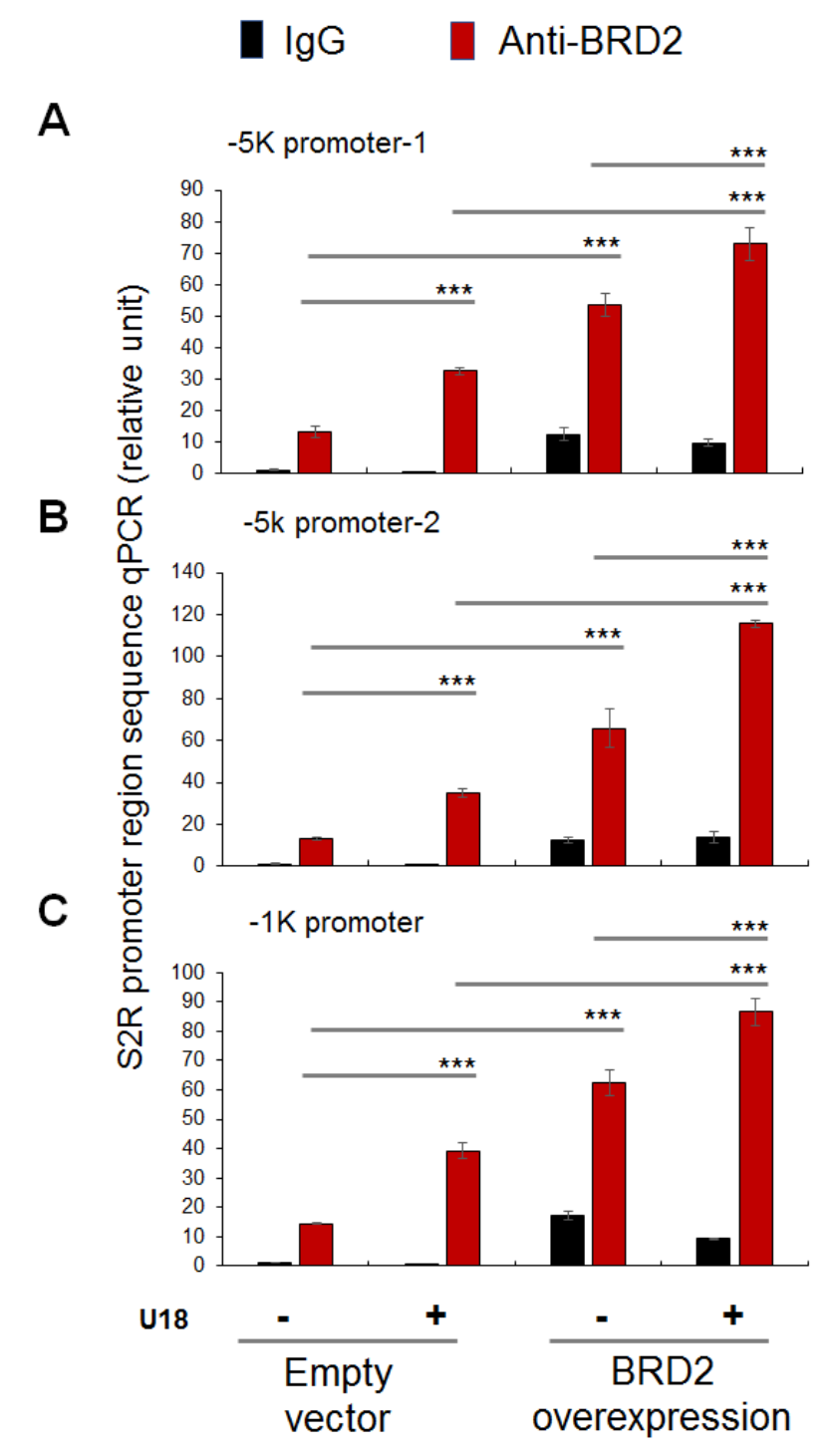

Figure S4. BRD2 occupies S2R gene promoter regions

ChIP-qPCR was performed as described in Figure 7B. Shown are results from all 3 experiments to detect BRD2 occupancy at 3 different S2R gene promoter regions, one of which (B) is presented in Figure 7B. The data indicate that all 3 experiments led to similar results. 

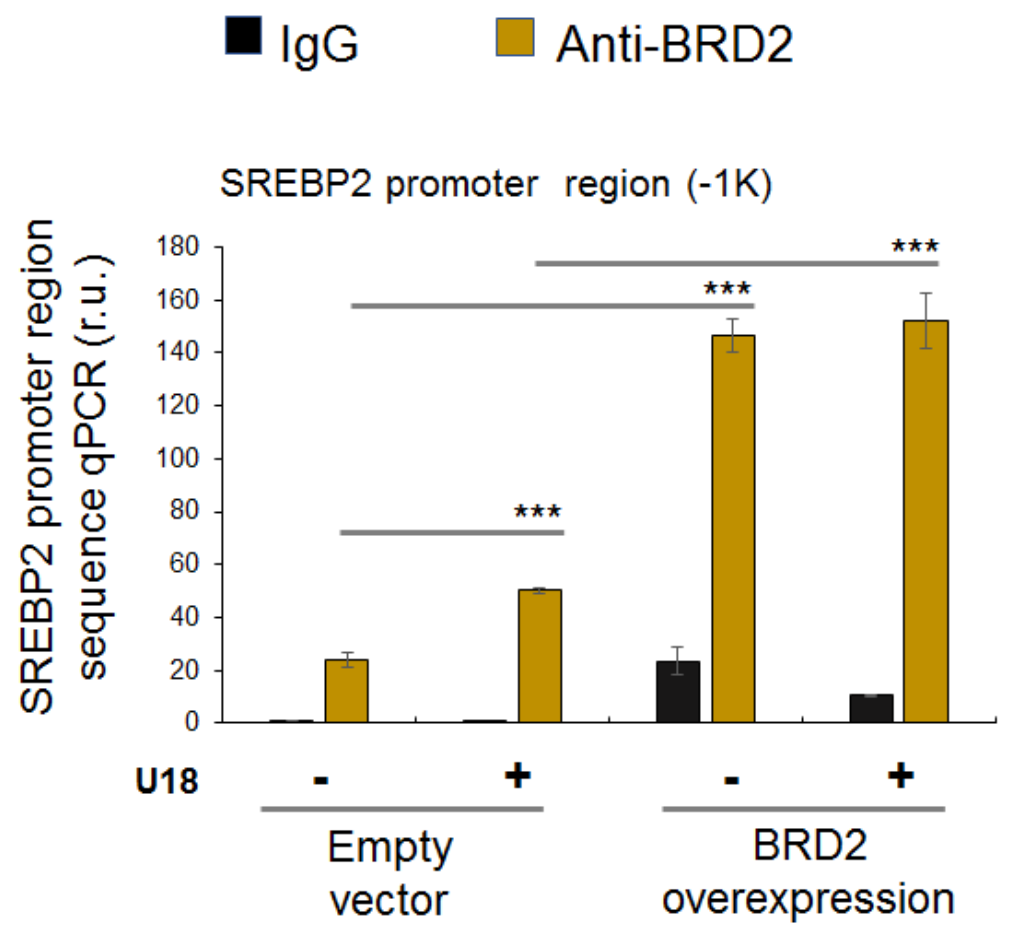

Figure S5. BRD2 antibody immunoprecipitates a SREBP2 gene promoter region

ChIP-qPCR was performed as described in Figure 7B. Shown is the experiment for the detection of BRD2 occupancy at the SREBP2 gene promoter. The data indicates that SREBP2 gene promoter (region) DNA was immunoprecipitated together with BRD2 by ChIP. 

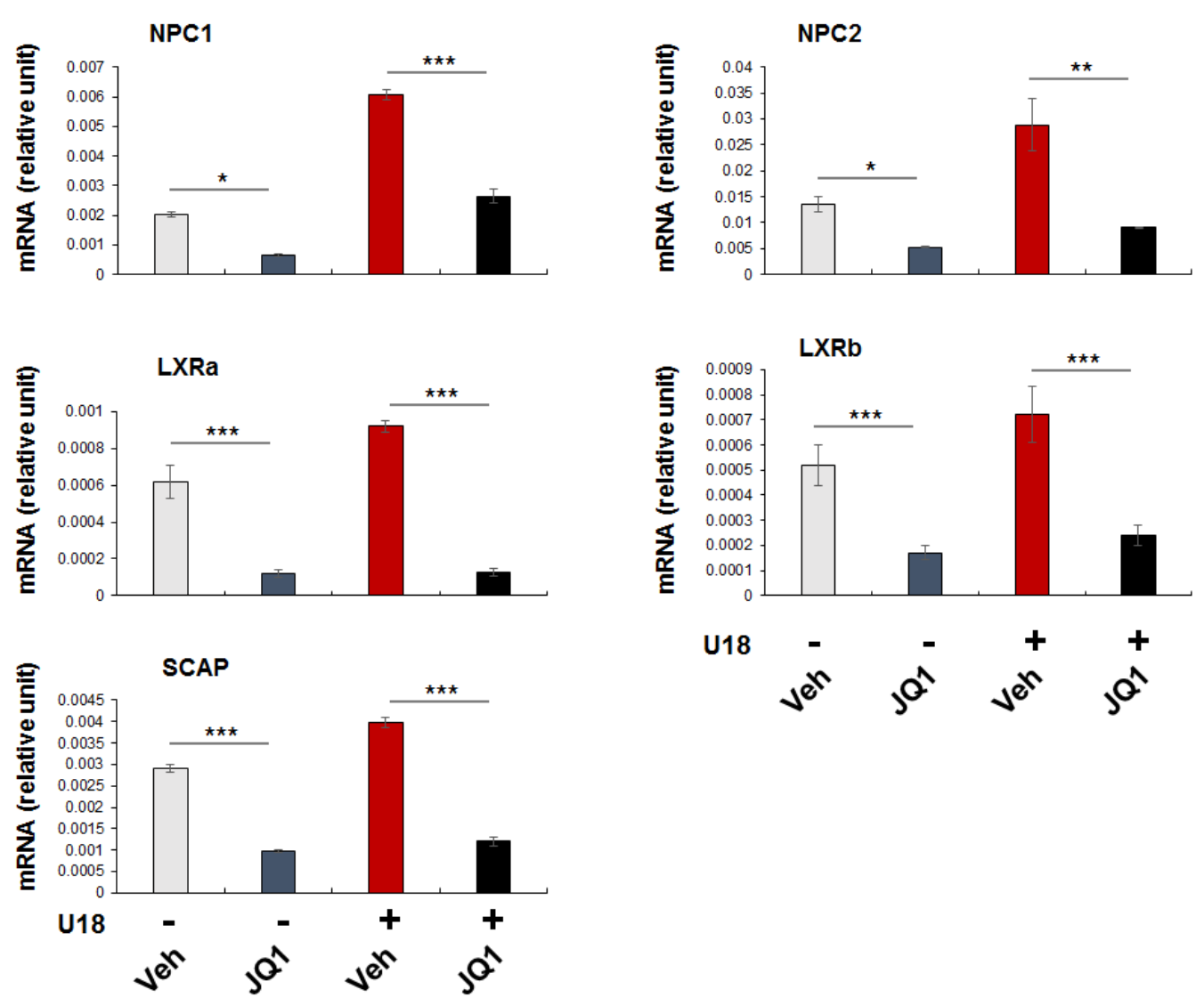

Figure S6. Pan-BETs inhibition attenuates the expression of SREBP target genes

Experiments were performed as described in Figure 1 except that several SREBP target gene mRNAs were assayed by qRT-PCR. Shown is each representative of two similar yet independent experiments. Statistics: Mean $\pm S D, n=3$. One-way ANOVA with Bonferroni posthoc test; ${ }^{*} \mathrm{P}<0.05,{ }^{* *} \mathrm{P}<0.01,{ }^{* * *} \mathrm{P}<0.001$. 


\section{Supplemental Tables}

Table S1. Primers used for qRT-PCR

\begin{tabular}{|l|l|l|}
\hline & Forward & Reverse \\
\hline BRD2 & GAGCCTGGACATCAACAAAT & GCAGGAAAGGACATAGCG \\
\hline BRD3 & ACGACATCATCAAGCACCCG & TTGGCAAACCTCATCTCAAACACG \\
\hline BRD4 & GTGGGAGGAAAGAAACAGGGAC & AGGAGGATTCGGCTGAGGG \\
\hline LXRa & GAGGTACAACCCTGGGAGTGAG & ATAGCAATGAGCAAGGCAAACT \\
\hline LXRß & GCTAACAGCGGCTCAAGAACTAAT & GCTCCGTGAAGTGGGCAAAG \\
\hline NPC1 & CCTTGTCTGGGTACGTTTG & AGGGCAGTGGCGTTATT \\
\hline NPC2 & GCGTCCCAGTTCCCTTTC & GTTGCCACTCCACCACC \\
\hline S2R & CAGTGGTTTCAAGGGACAAAG & GAATGGGATGAGTAAGTAGGG \\
\hline SCAP & GACTCTGACCGCAAACAAGG & GGGACAAAGGTGAACGAAATAC \\
\hline SREBP1 & CTTTGCCGACCCTGGTGAGT & ATGGCGTTGTGGGCTGTGC \\
\hline SREBP2 & ATTGTCCTGAGCGTCTTTGTG & CAGGCAGGTTTGTAGGTTGC \\
\hline GAPDH & TGCACCACCAACTGCTTAGC & GGCATGGACTGTGGTCATGAG \\
\hline
\end{tabular}

Table S2. Oligonucleotide sequences for shRNAs

\begin{tabular}{|l|l|l|}
\hline & Sense & Anti-sense \\
\hline BRD3-1 & CCGGGCTGATGTTCTCGAATTG & AATTCAAAAACTGATGTTCTCGAATT \\
& CTACTCGAGTAGCAATTCGAGAA & GCTACTCGAGTAGCAATTCGAGAACAT \\
CATCAGCTTTTG & CAGC \\
\hline BRD3-2 & CCGGGAGATATGTCAAGTCTTGT & AATTCAAAAAGAGATATGTCAAGTCTT \\
& TTCTCGAGAAACAAGACTTGACA & GTTTCTCGAGAAACAAGACTTGACATA \\
& TATCTCTTTTTG & TCTC \\
\hline BRD4-1 & CCGGCAGTGACAGTTCGACTGA & AATTCAAAAAACAGTGACAGTTCGACT \\
& $\begin{array}{l}\text { TGACTCGAGTCATCAGTCGAACT } \\
\text { GTCACTGTTTTTTG }\end{array}$ & GATGACTCGAGTCATCAGTCGAACTGT \\
& CACTG \\
\hline BRD4-2 & CCGGCCTGGAGATGACATAGTC & AATTCAAAAACCTGGAGATGACATAGT \\
& TTACTCGAGTAAGACTATGTCAT & CTTACTCGAGTAAGACTATGTCATCTC \\
CTCCAGGTTTTTG & CAGG \\
\hline SREBP2 & CCGGCCTCAGATCATCAAGACA & AATTCAAAAACCTCAGATCATCAAGAC \\
& GATCTCGAGATCTGTCTTGATGA & AGATCTCGAGATCTGTCTTGATGATCT \\
& TCTGAGGTTTTTG & GAGG \\
\hline Scram- & CCGGCCTAAGGTTAAGTCGCCC & AATTCAAAAACCTAAGGTTAAGTCGCC \\
bled & TCGCTCGAGCGAGGGCGACTTA & CTCGCTCGAGCGAGGGCGACTTAACC \\
& ACCTTAGGTTTTTG & TTAGG \\
\hline
\end{tabular}


Table S3. Antibodies for Western blotting

\begin{tabular}{|l|l|l|l|}
\hline Antibody & Company & Catalog number & Dilution \\
\hline BRD2 & Proteintech & $22236-1-A P$ & $1: 1000$ \\
\hline BRD3 & Proteintech & $11859-1-A P$ & $1: 1000$ \\
\hline BRD4 & Abcam & Ab75898 & $1: 1000$ \\
\hline SREBP1 & Santa cruz & Sc-13551 & $1: 1000$ \\
\hline SREBP2 & BD Biosciences & 557037 & $1: 200$ \\
\hline S2R & Novus & NBP1-30436 & $1: 200$ \\
\hline FLAG & Sigma-aldrich & F3165 & $1: 2000$ \\
\hline GAPDH & $\begin{array}{l}\text { Cell Signaling } \\
\text { Technologies }\end{array}$ & $2118 S$ & $1: 3000$ \\
\hline$\beta$-Actin & Abcam & ab6276 & $1: 3000$ \\
\hline
\end{tabular}

Table S4. Primers for ChIP-qPCR

\begin{tabular}{|l|l|l|l|}
\hline & & Forward primer & Reverse primer \\
\hline \multirow{2}{*}{$\begin{array}{l}\text { S2R } \\
\text { gene } \\
\text { promoter }\end{array}$} & $\begin{array}{l}\text { Region-1 } \\
(-5 \mathrm{~K} \text { bp } \\
\text { from TSS })\end{array}$ & ATAGAGGCAATTGGGGGCG & AATGCACCCACCCTTCT \\
\cline { 2 - 4 } & $\begin{array}{l}\text { Region-2 } \\
(-5 \mathrm{~K})\end{array}$ & GGGGTAGGGTGGCCTTCAGCC & AATGCACCCACCCTTCT \\
\cline { 2 - 4 } & $\begin{array}{l}\text { Region-3 } \\
(-1 \mathrm{~K})\end{array}$ & GCCCTCTGCTCGCCATCAAAG & GGAAGGAATGCAGCTTGGACT \\
\hline & & & \\
\hline $\begin{array}{l}\text { SREBP2 } \\
\text { gene } \\
\text { promoter }\end{array}$ & $\begin{array}{l}\text { A region } \\
\text { at -1K bp } \\
\text { from TSS }\end{array}$ & GGGGAGGGACCTCACTATGC & AGCCAATGGGCGAGCGAAGC \\
\hline
\end{tabular}

